\title{
Synthesis of semiconductor nanoparticles and characterization of physical and optical properties
}

\author{
Mikala Shremshock \\ West Virginia University
}

Follow this and additional works at: https://researchrepository.wvu.edu/etd

\section{Recommended Citation}

Shremshock, Mikala, "Synthesis of semiconductor nanoparticles and characterization of physical and optical properties" (2009). Graduate Theses, Dissertations, and Problem Reports. 2803.

https://researchrepository.wvu.edu/etd/2803

This Thesis is protected by copyright and/or related rights. It has been brought to you by the The Research Repository @ WVU with permission from the rights-holder(s). You are free to use this Thesis in any way that is permitted by the copyright and related rights legislation that applies to your use. For other uses you must obtain permission from the rights-holder(s) directly, unless additional rights are indicated by a Creative Commons license in the record and/ or on the work itself. This Thesis has been accepted for inclusion in WVU Graduate Theses, Dissertations, and Problem Reports collection by an authorized administrator of The Research Repository @ WVU. For more information, please contact researchrepository@mail.wvu.edu. 


\title{
Synthesis of Semiconductor Nanoparticles and Characterization of Physical and Optical Properties
}

\author{
by \\ Mikala Shremshock \\ Thesis submitted to the Eberly College of Arts and Sciences \\ at West Virginia University \\ in partial fulfillment of the requirements for \\ the degree of \\ Master of Science \\ in \\ Chemistry \\ R. Lloyd Carroll, Ph.D., Chair \\ Jeffrey Petersen, Ph.D. \\ Alan Stolzenberg, Ph.D. \\ C. Eugene Bennett Department of Chemistry \\ Morgantown, West Virginia \\ 2009
}

Keywords: quantum dot, nanorod, nanoparticle, semiconductor, biomimetic Copyright 2009 Mikala Shremshock 


\section{Abstract \\ Synthesis of Semiconductor Nanoparticles and Characterization of Physical and Optical Properties}

\section{Mikala Shremshock}

Nanoparticles are of great interest to a broad scientific community. Because of quantum effects, nanoscale materials exhibit many unique properties that may be exploited for biomedical, defense, and energy applications. Extensive synthetic effort is described for II-VI semiconductor materials. The materials were morphologically confined to zero- or onedimensional structures. The nanoparticles discussed herein present similar chemical, optical, and physical properties to previously synthesized materials; however, the processes used to obtain these particles are more environmentally benign and use safer chemicals for the researcher and the laboratory environment. These nanomaterials have been extensively characterized to ascertain high quality optical, morphological, and electronic properties as well as viability with biomolecular and cellular conjugation assays.
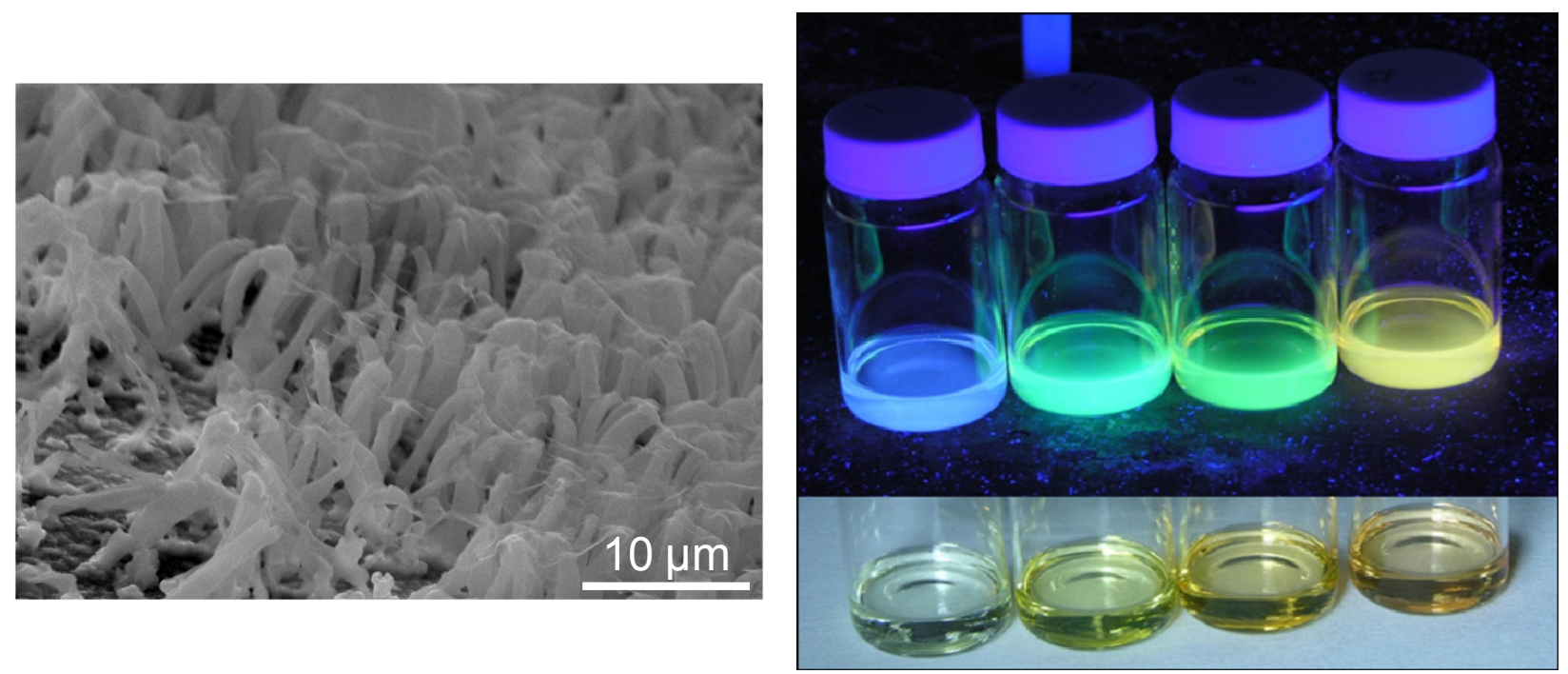


\section{Acknowledgements}

I would like to thank my research advisor, Dr. R. Lloyd Carroll, for his support and guidance during my time in graduate school. I am also appreciative to his family for their understanding and generosity. I would like to thank the other members of my committee, Dr. Jeffrey Petersen and Dr. Alan Stolzenberg for their support and enlightening discussions. The other professors in the WVU Chemistry department have been helpful and accommodating wherever possible. Without the support of Barbara, Sheri, JR, Kevin, Mike, Eze, Allen, Sherman, and Phil, none of this work could have ever been possible.

I would also like to thank professors and staff throughout the university who have helped me find my way around their buildings, trained me on a myriad of instruments and techniques. I would like to especially thank Kolin Brown, Eric Shires, Dr. Andy Woodworth, Cameron Keenan, Dr. David Lederman, Dr. Chad Junkermirer, Dr. Vin Berry, Dr. Pete Gannett, and Jarod Kabulski. I would also like to thank the entire Nano Class where so many strong collaborations and brilliant ideas were formed!

I would like to thank my fellow lab mates, especially, Shengrong Ye, who has put up with me the longest. Also, a good deal of work and fun was had with the summer undergraduate researchers and especially those who stuck around throughout the semesters. Of special importance are Dru Burns, a WVU student who aided in my early quantum dot research and Cathy Yee, an REU student from University of Connecticut who assisted greatly in the semiconductor nanorod work.

I would like to thank my family and friends who were kind enough to support me and listen to me complain even when they had no idea what I was talking about. In particular, Jessica Gutshall and Stephanie Archer have been stalwart friends through my many personal and professional successes and failures. 


\section{List of Figures}

Figure 1-1. Electromagnetic spectrum correlated with QD material..................................2

Figure 1-2. Conceptual representation showing possible dimensions of confinement and extension for nanostructured systems. .............................................................

Figure 2-1. CdSe QDs under ultraviolet (top) and ambient (bottom) light. Size ranges from $\sim 2$ $\mathrm{nm}$ (left) to $\sim 4 \mathrm{~nm}$ (right) in diameter.

Figure 2-2. Absorbance (left) and PL spectra (right) of CdSe QDs in chloroform solution synthesized with increasing growth times. (Spectra are vertically shifted for clarity.) 9

Figure 2-3. Fluorescence spectra of three distinct $\mathrm{CdSe}$ core reactions quenched after identical growth time. $\Delta \lambda=3 \mathrm{~nm}$ across all three solutions.

Figure 2-4. Energy dispersive x-ray spectroscopy of CdSe/ZnS core/shell QDs verifying the qualitative elemental composition.

Figure 2-5. PL spectra of CdSe cores grown for 45 seconds, purified, and redispersed in chloroform and the same CdSe cores after ZnS shell addition ( 4 monolayers) and purification.

Figure 2-6. TEM of (a) CdSe cores synthesized for $45 \mathrm{sec}$ and (b) of same CdSe cores with $\sim 4$ monolayers of ZnS. Insets show histogram and Gaussian fit of particle size...........15

Figure 2-7. XPS spectrum of CdSe/ZnS QDs............................................................. 16

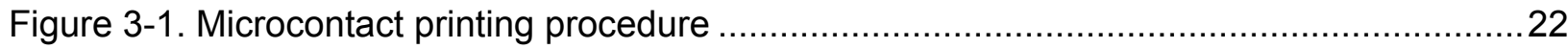

Figure 3-2. Infrared spectra of (a) neat TOPO and (b) TOP surfacted QDs.........................24

Figure 3-3. Time series of IR spectra of ligand exchange of TOP for MPA. .........................25

Figure 3-4. Confocal microscopy images of QD emission (DAPI filter; red-channel) and FITC emission (FITC filter; green-channel).

Figure 3-5. Fluid AFM images of (a) BSA stamp and (b) BSA stamp with MPA-QDs. Height traces are of BSA stamp before (green) and after (red) QD conjugation.

Figure 3-6. Confocal image of cells after conjugation with Ab-QDs and immunolabeling. a) red channel showing $600 \mathrm{~nm}$ QDs, b) green channel showing FITC (514nm), and c) colocalization of red and green produce yellow.

Figure 4-1. Schematic of CdS nanorod reaction and growth system.................................. 34

Figure 4-2. Photograph of actual reaction system used to produce CdS nanostructures...........36

Figure 4-3. SEM images of CdS rods grown in PCTE membrane before (left) and after (right)

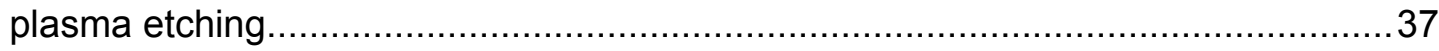

Figure 4-4. SEM images of CdS tubes (left) and rods (right) ...................................... 39 
Figure 4-5. Optical micrographs of CdS macro crystals. ........................................... 40

Figure 4-6. EDS spectrum of CdS crystals. ...................................................................... 41

Figure 4-7. XRD spectrum of CdS crystals with comparison to CdS cubic and hexagonal peaks.

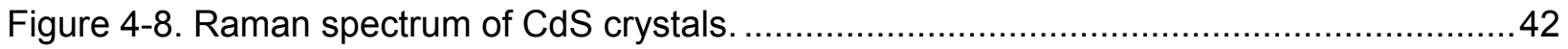

Figure 4-9. Schematic of CdS tube and rod formation supported with SEM images of actual

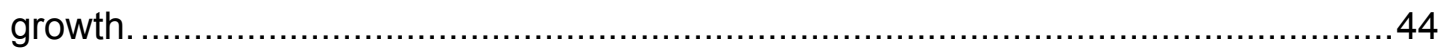




\section{List of Schemes}

Scheme 2-1. Conceptual schematic of the synthesis of CdSe quantum dot cores with organic surfactant coating.

Scheme 2-2. Conceptual schematic of the synthesis of addition of $\mathrm{ZnS}$ shell with similar organic surfactant coating.

Scheme 3-1. A generalized scheme of carbodiimide (EDC) and hydroxy succinimide (NHS) coupling of carboxylate-terminated molecules (1) with amine-terminated molecules (2).

Scheme 3-2. Conceptual schematic of hydrophobic-to-hydrophilic ligand exchange...............21

Scheme 3-3. Conceptual schematic of polymeric encapsulation of QD. .............................21

Scheme 4-1. Reaction used to produce hydrogen sulfide gas for subsequent reaction............34

Scheme 4-2. Reaction that occurs in the membrane pores to produce CdS rods.....................35 


\section{List of Tables}

Table 2-1. Comparison of size determinations for three QD CdSe cores synthesized with increasing growth times based on experimental data fit, effective mass model calculation, and TEM observations.

Table 3-1. Comparison of QDs and organic fluorescent dyes for biological labeling experiments. 18

Table 4-1. Comparison of experimental conditions and observed nanostructures. 43 


\section{Contents}

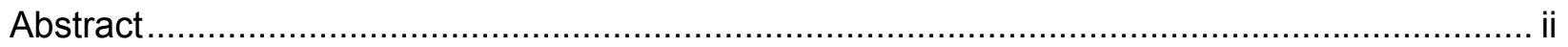

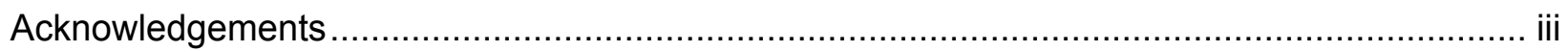

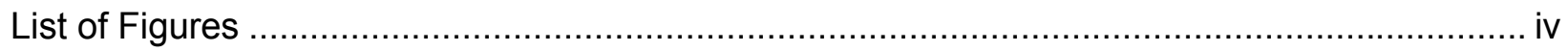

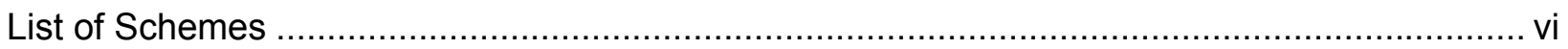

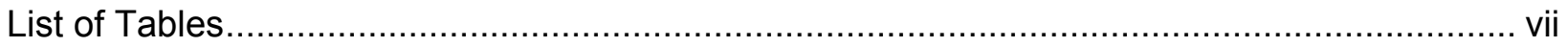

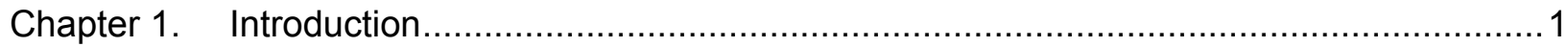

Chapter 2. Semiconductor Quantum Dot Synthesis and Characterization ...........................3

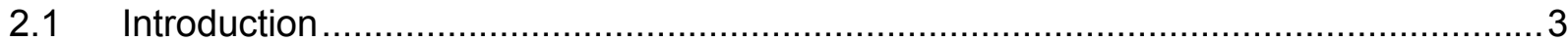

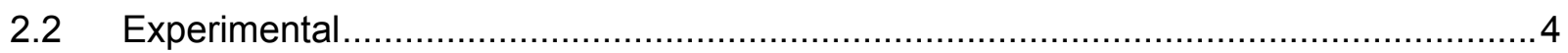

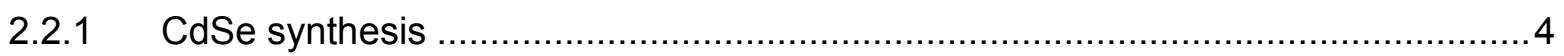

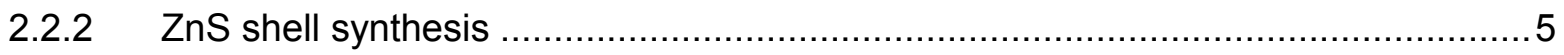

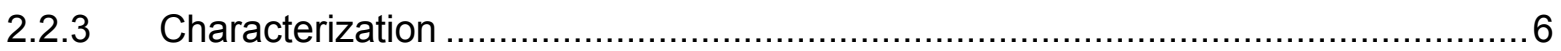

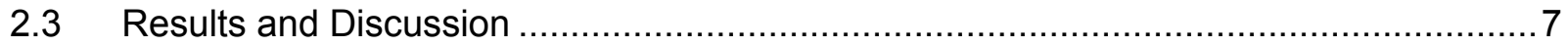

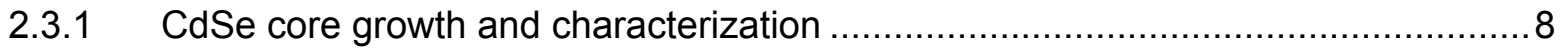

2.3.2 ZnS shell growth and characterization ........................................................ 10

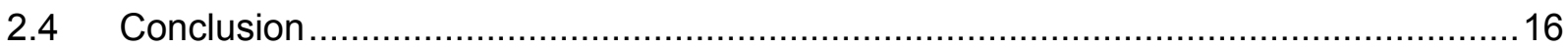

Chapter 3. QD surface modification and functionalization ........................................ 18

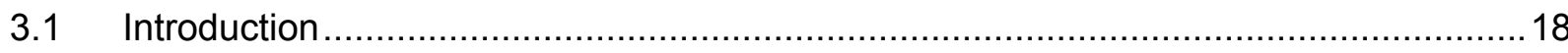

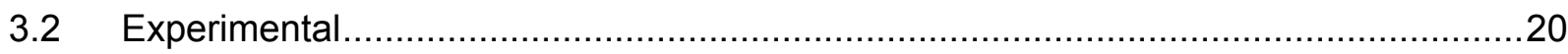

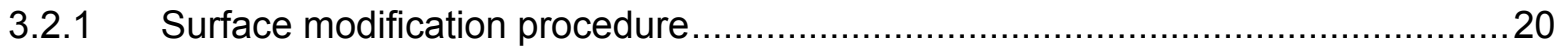

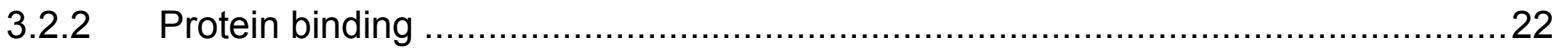

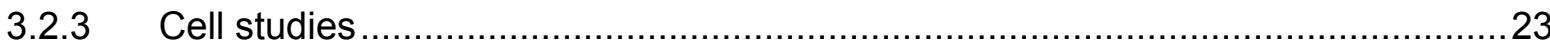

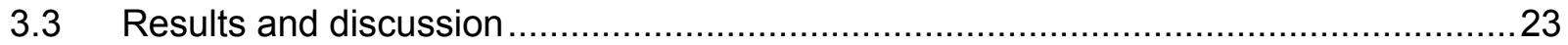

3.3.1 Surface modification characterization .....................................................23

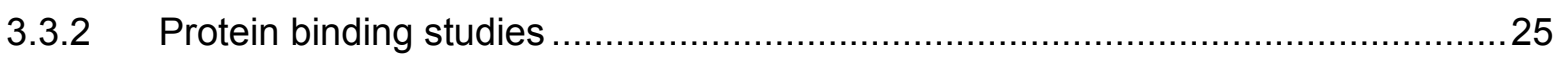

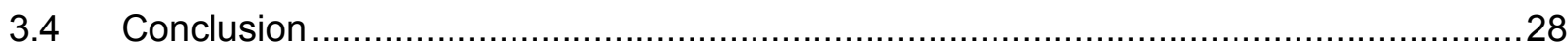

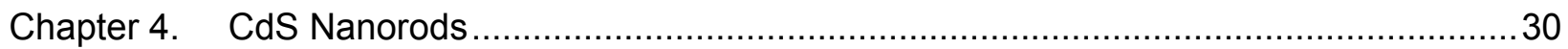




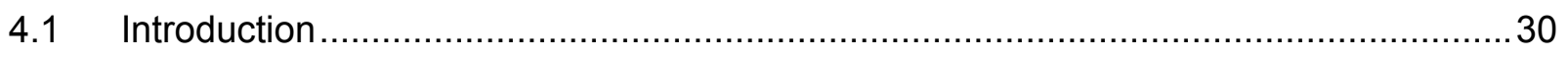

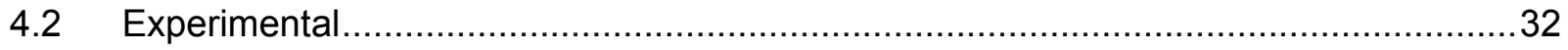

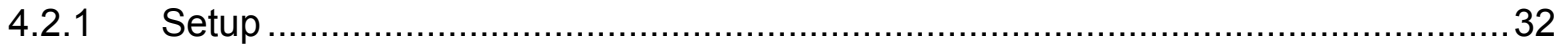

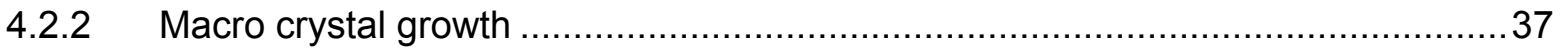

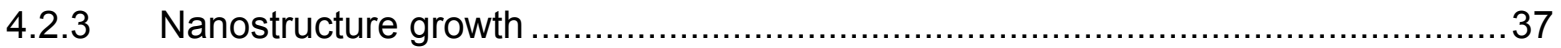

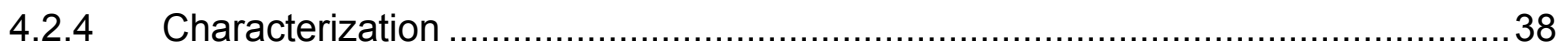

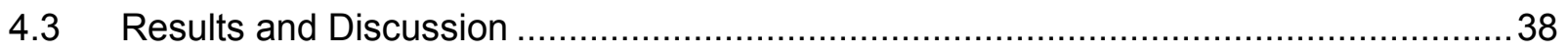

4.3.1 Macro crystal growth and characterization ............................................. 40

4.3.2 Nanostructure growth and characterization.............................................43

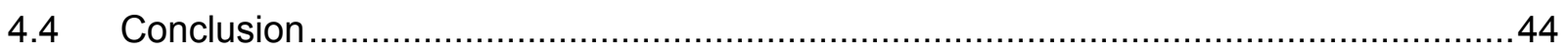

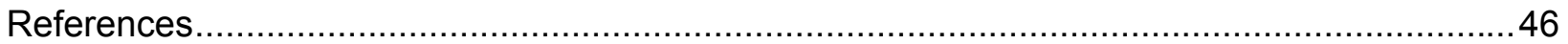




\section{Chapter 1. Introduction}

Nanoscale materials harbor great potential in a variety of fields. Nano-phenomena have already been harnessed to develop many new applications in medical diagnostics and treatments, energy, lighting, and homeland security. They have demonstrated great utility in biomedical imaging, ${ }^{1-6}$ engineering (photonic crystals), ${ }^{7,8}$ photovoltaics, ${ }^{9,10}$ solid state lighting, ${ }^{11-}$ ${ }^{14}$ quantum computing, ${ }^{15-17}$ security inks and devices, ${ }^{18}$ industrial dyes, ${ }^{19}$ and chemical sensors. $^{20-23}$

The term "nanoparticle" describes a piece of material with any shape that has at least one dimension less than $100 \mathrm{~nm}$. In addition, they typically exhibit properties different from the bulk substance such as fluorescence, conductivity, color change, or reactivity. These materials have been exploited for art or scientific pursuits for centuries, ${ }^{24-26}$ yet many properties are still being discovered and characterized. New materials, new synthetic approaches, and new analytical techniques continue to elucidate the variety of structures and functions that nanoscale materials can exhibit.

While nanomaterials can consist of almost any element, metals and semiconductors have proven to have the most diverse applications (Figure 1-1). This work primarily investigates cadmium chalcogenides such as cadmium selenide and cadmium sulfide. These and other II-IV type semiconductors have been well studied. ${ }^{14,27-49}$ Nanoscale semiconductor materials exhibit unique photophysical properties such as shifts in absorption range and photoluminescence. ${ }^{50}$ These properties arise because of quantum effects produced by confinement of the band structure and tuning of the band edge features of particular semiconductor materials. ${ }^{51}$ 


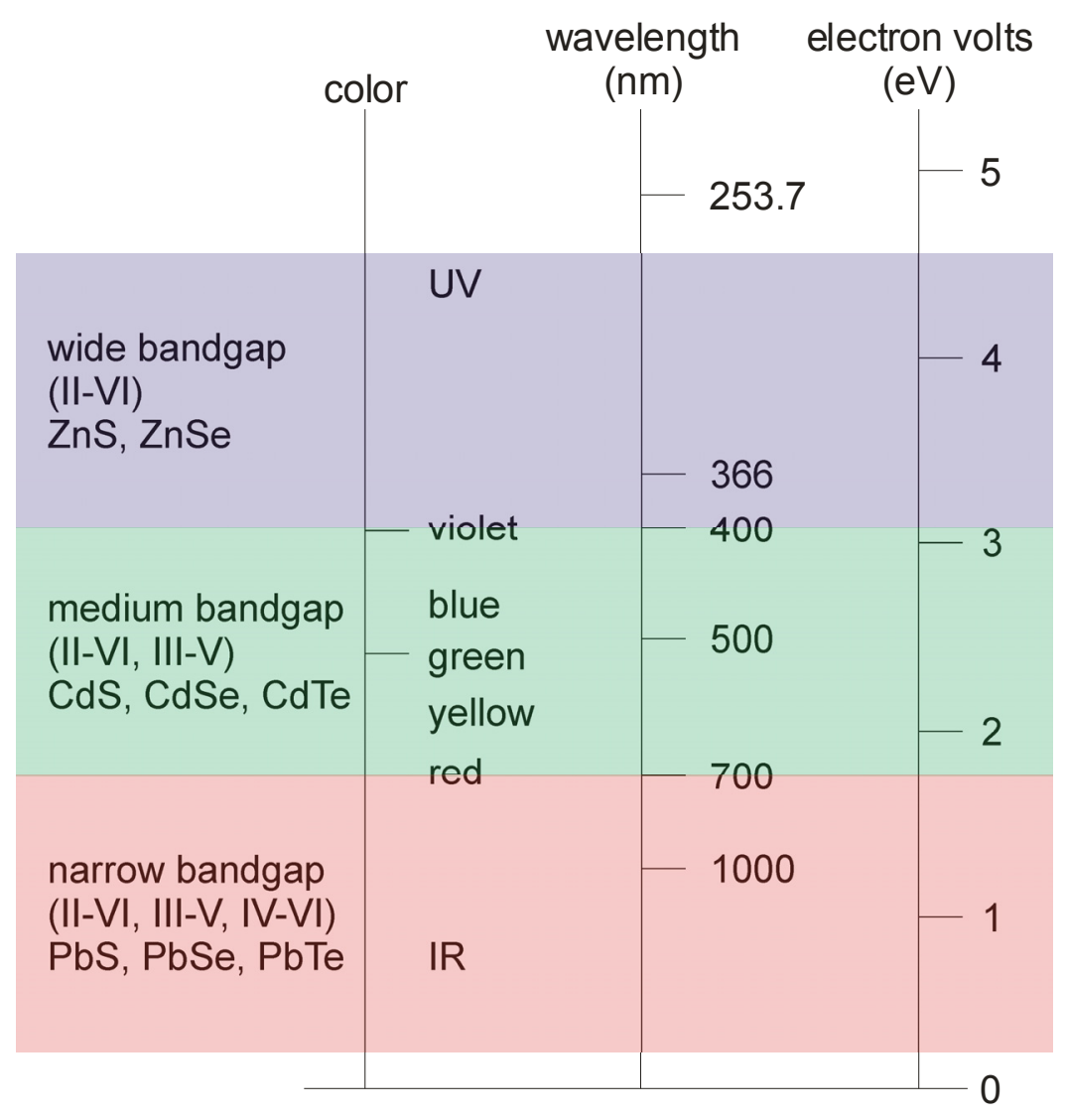

Figure 1-1. Electromagnetic spectrum correlated with QD material.

In this work, two primary morphologies of nanoparticles were synthesized and characterized - spherical nanoparticles known as quantum dots (QDs) and anisotropic nanorods and tubes which are considered as one-dimensional materials. These structures have been previously synthesized using a variety of methods including molecular epitaxy, ${ }^{52,53}$ templating, ${ }^{54-56}$ organometallic, ${ }^{39,57,58}$ solvothermal, vapor-liquid-solid, ${ }^{59-61}$ and seeded approaches.

Structures can be classified by the number of dimensions in which their size is confined (Figure 1-2). A structure confined in all three dimensions, $x, y$, and $z$, is called a nanoparticle or quantum dot. If a structure is confined in two dimensions, it is a nanowire or nanorod. A thin film may theoretically extend for meters in the $x$ and $y$ directions, but is confined in the $z$ direction. 
For analysis and characterization, particles are typically considered as spheres or rods although they are usually multifaceted due to their crystalline structure.
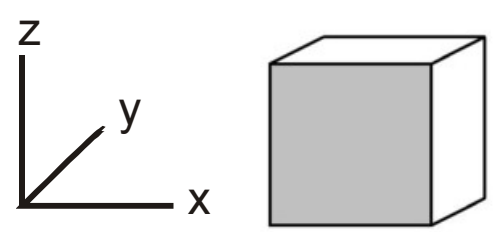

bulk

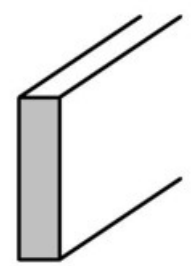

thin film

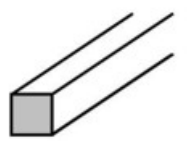

nanowire

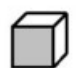

nanoparticle $1 \mathrm{D}$
OD

Figure 1-2. Conceptual representation showing possible dimensions of confinement and extension for nanostructured systems.

\section{Chapter 2. Semiconductor Quantum Dot Synthesis and Characterization}

\subsection{Introduction}

Quantum dots (QDs) are spherical nanoparticles made of a semiconducting material that experience quantum confinement due to their small size (Figure 2-1). Because QD dimensions are smaller than the Bohr exciton radius, electrons excited from the valence band are confined to lower energy. This is known as quantum confinement. QDs are of great importance for biological, engineering, and chemical applications. ${ }^{62,63}$ In particular, core/shell type materials have greater potential impact due to their increased stability and quantum efficiency and reduced susceptibility to solution degradation thereby reducing cytotoxicity. ${ }^{37,64} \mathrm{CdSe}$ cores alone are susceptible to oxidation producing $\mathrm{CdO}$ and $\mathrm{SeO}_{2} \cdot{ }^{37,65,66}$ While environmentally benign syntheses have been developed for CdSe core QDs, ${ }^{67,68} \mathrm{ZnS}$ shell synthesis has, until recently, required the use of toxic and pyrophoric materials that require careful handling and disposal limiting the accessibility of such materials for extensive research exploration. ${ }^{46,69}$ Here, we 
present the characterization and analysis of CdSe/ZnS core/shell QDs synthesized using green chemistry principles. The data demonstrate that the QDs are of high quality (monodisperse, photostable, reproducible). The synthesis is reasonably simple and reproducible. The resulting nanomaterials can be readily modified for use in further scientific or technical applications. Such results should provide greater opportunities for research and exploration of quantum dot applications.

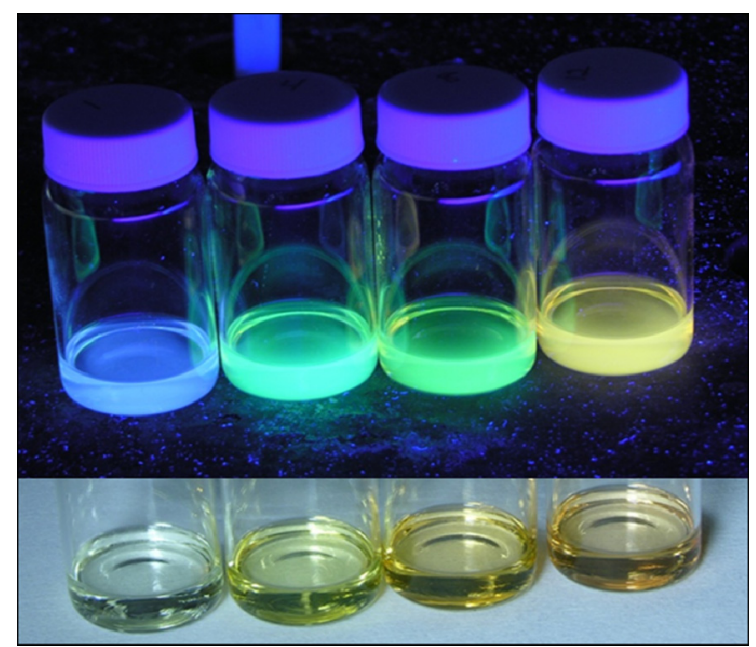

Figure 2-1. CdSe QDs under ultraviolet (top) and ambient (bottom) light. Size ranges from $\sim 2 \mathrm{~nm}$ (left) to $\sim 4 \mathrm{~nm}$ (right) in diameter.

\subsection{Experimental}

All chemicals were used as received without further purification. Cadmium oxide (99.998\%) and selenium (99.999\%) were purchased from Alfa Aesar. Technical grades of octadecene (ODE), trioctylphosphine (TOP), and 1-hexadecylamine (HDA) were purchased from Aldrich. Tri-n-octylphosphine oxide (TOPO; 99\%), zinc stearate, sulfur (99.999\%), and mercaptopropionic acid (MPA) were obtained from Acros. Oleic acid and other solvents were from Fisher.

\subsubsection{CdSe synthesis}


CdSe QD core synthesis was derived from previously reported methods. ${ }^{40,42}$ No effort was made to exclude air from the reaction. In a typical synthesis (Scheme 2-1), $130 \mathrm{mg} \mathrm{CdO}$ was dissolved in $100 \mathrm{ml}$ ODE and $6 \mathrm{ml}$ oleic acid in a $250 \mathrm{ml}$ round bottom flask. TOP(Se) solution was prepared by dissolving $60 \mathrm{mg}$ Se in $0.8 \mathrm{ml}$ TOP at room temperature. The CdO solution was heated at $200^{\circ} \mathrm{C}$ until a clear golden-yellow solution was observed. The selenium solution was then rapidly injected into the cadmium solution. When the QDs reached the desired size, the reaction was quenched by pouring the solution into a clean vial immersed in a slurry of ice, water, and methanol at $-15^{\circ} \mathrm{C}$ for 15 minutes or by pouring $100 \mathrm{ml}$ of room temperature ODE directly into the reaction mixture. The nanoparticles were purified by precipitation with methanol and redispersed in chloroform.

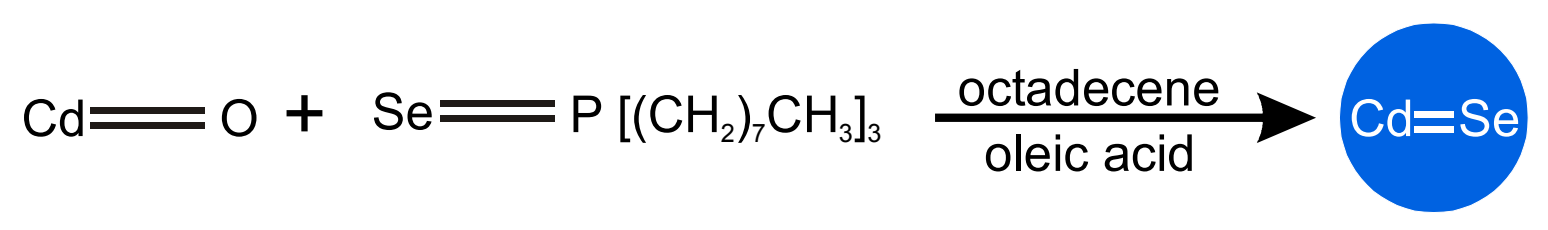

Scheme 2-1. Conceptual schematic of the synthesis of CdSe quantum dot cores with organic surfactant coating.

\subsubsection{ZnS shell synthesis}

In the ZnS shell synthesis (Scheme 2-2), CdSe cores $(50 \mathrm{mg}$ ) in chloroform were heated at $65^{\circ} \mathrm{C}$ under argon flow until all solvent was removed. $2 \mathrm{ml}$ each of HDA and TOPO was added and the temperature was gradually increased to $190^{\circ} \mathrm{C}$. An equimolar mixture of zinc stearate and elemental sulfur in $2.5 \mathrm{ml}$ of toluene and $2.5 \mathrm{ml}$ of TOP respectively was transferred to the reaction flask at a rate of $0.1 \mathrm{ml} / \mathrm{min}$ by syringe pump. Following injection, the solution was heated for an additional hour and then cooled. The presence of high molecular weight ligands such as stearate, HDA, and TOP caused the reaction solution to solidify on cooling to room temperature. The addition of 1-2 $\mathrm{ml}$ of 2 -butanol to the reaction solution after 
cooling to below the boiling point of butanol mitigated this problem and allowed purification by precipitation and centrifugation. The particles were precipitated by addition of methanol and were redispersed in chloroform for characterization and further experimentation.

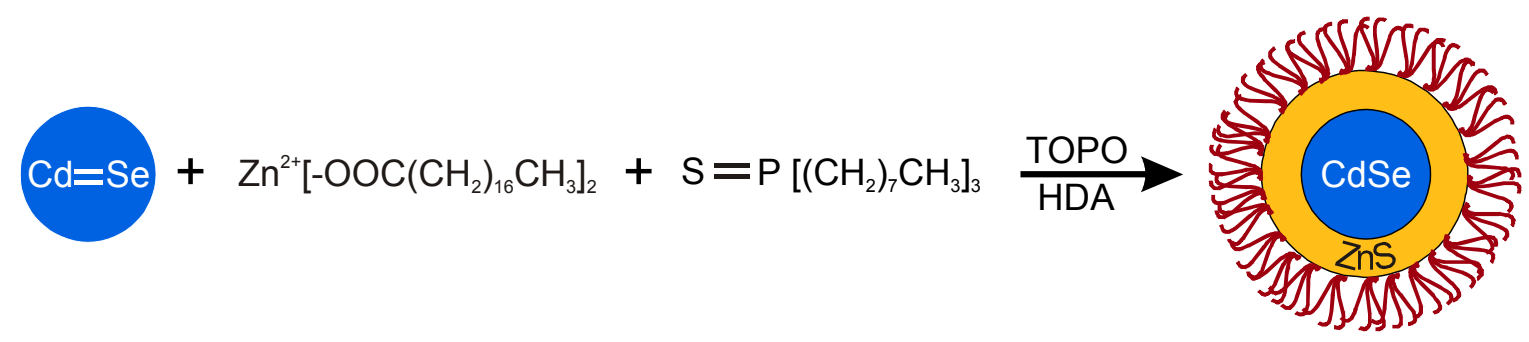

Scheme 2-2. Conceptual schematic of the synthesis of addition of ZnS shell with similar organic surfactant coating.

\subsubsection{Characterization}

Ultraviolet-visible absorption spectroscopy: Absorption spectra were obtained on a Varian Cary 50 Bio Ultraviolet-Visible Spectrophotometer. Samples were prepared in chloroform or water and analyzed in $1 \mathrm{~cm}$ quartz cuvettes.

Photoluminescence spectroscopy: PL spectra were obtained on a Horiba Jobin-Yvon Fluorolog-3 spectrophotometer with a xenon lamp source. Slits were set to $5 \mathrm{~nm}$ with an integration time of $0.1 \mathrm{~s}$. Solutions were prepared in chloroform or water and analyzed in $1 \mathrm{~cm}$ quartz cuvettes. Sample absorbance was typically 0.1 or lower to prevent reabsorption effects.

Infrared spectroscopy: Infrared spectroscopy was performed on a Perkin-Elmer ATRFTIR with $4 \mathrm{~cm}^{-1}$ resolution. 8-scan averaging was performed to improve signal/noise ratio. Liquid samples in chloroform were deposited dropwise to form a thin film on the zinc selenide ATR sampling crystal. Aqueous samples were prepared as thin films on glass cover slides. X-ray photoelectron spectroscopy: XPS was performed on a Physical Electronics VersaProbe 5000 with a monochromated aluminum source using ion neutralization. Samples 
were prepared by evaporation of organic based solutions or powder deposition on cleaned silicon wafers. Data were analyzed using PHI MultiPak.

Electron microscopy: Scanning electron microscopy was performed and electron dispersive x-ray spectra were obtained on a JEOL JSM6490-LVwith an INCA Energy SiLi detector. Samples were prepared by evaporating a thin film of QDs on HOPG. TEM was performed on a JEOL 100CX II. Samples were prepared from organic and aqueous solutions deposited on carbon TEM grids. Particle size data was determined by analyzing the binary images in ImageJ to obtain a histogram with a Gaussian fit. Number of measurements (n) was at least 100.

\subsection{Results and Discussion}

QDs have become important research materials in a variety of areas because of their unique and tunable optoelectronic properties, such as fluorescence and tunable bandgap size. II-VI semiconductor nanomaterials have been well studied because of their band gap emission of visible spectrum light. ${ }^{47,57,64,70,71}$ QDs are in development for use in solid state lighting and photovoltaics, security and identification systems, and biomolecular targeting and delivery. QDs have been widely used in biological labeling and microscopy studies because of their benefits over traditional organic fluorophores. QDs typically have brighter emission, wider excitation wavelength windows, and longer photostability. ${ }^{32,57}$ QDs are being further explored for use as biomedical diagnostic and therapeutic delivery agents, but cytotoxicity has been a hindrance. ${ }^{37,65,72,73}$ Core/shell type QDs are of great benefit due to their increased stability and quantum efficiency. Two-material QDs also experience less rapid degradation and decreased toxicity.

Here, we have synthesized our own CdSe/ZnS core/shell QDs using a more environmentally benign reaction. This QD synthesis eliminates the need for extreme 
temperatures or glove box techniques and avoids unstable and hazardous compounds used in typical nanoparticle shell syntheses, while still producing QDs of uniform size and spectral properties. This work demonstrates a user-friendly, high-yield route to QDs that can be readily functionalized and modified.

\subsubsection{CdSe core growth and characterization}

Dissolving cadmium oxide in the presence of oleic acid and other high boiling point solvents produces a cadmium oleate precursor. Mixing with TOP-Se at $220^{\circ} \mathrm{C}$ resulted in an initially translucent yellow solution, which changed to red over several minutes. After 10 minutes, no further apparent color changes were observed. Aliquots were frequently removed throughout the reaction (starting immediately after combination and continuing for $\sim 5$ minutes), quenched in iced methanol or room temperature ODE, and precipitated by the addition of methanol. Upon resuspension in chloroform, the photophysical properties of the suspensions were analyzed by absorbance and photoluminescence (PL) measurements (Figure 2-2). Emission spectra were collected using excitation at $480 \mathrm{~nm}$. Increasing growth time correlates with increasing absorption and emission wavelength (decrease in energy) consistent with increasing nanoparticle size. ${ }^{50}$ Absorbance spectra show shifts in band edge from bulk CdSe as determined from the local maxim of the absorbance peak wavelength. As synthesis time increases, QDs show decreased band gap energy consistent with decreased electron confinement in the particle due to increasing diameter. PL spectra show the full-width at halfmaximum (FWHM) to be $30 \mathrm{~nm}$ indicative of a highly monodisperse solution ( $5 \%$ size distribution). ${ }^{74}$ As reaction time is increased, both absorbance and emission peaks shift to longer wavelengths consistent with increasing QD size, decreasing quantum confinement, and decreasing bandgap energy. Absorbance measurements show that the band edge varies from $0.48 \mathrm{eV}$ for the longest reaction times to $0.56 \mathrm{eV}$ for shorter reaction times. This confirms that 
smaller nanoparticles with high-energy absorbances are initially formed and that particle diameter and reaction time have a direct linear relationship. PL measurements confirm fluorescence emissions in the visible range as expected from confinement in CdSe QDs. The emission wavelength increases as reaction time increases, consistent with increasing size and variable band gap of these particles. The narrow emission peaks (FWHM $\sim 30 \mathrm{~nm}$ ) indicate consistently monodisperse particles as compared with published results of previous syntheses. ${ }^{14,42,57}$

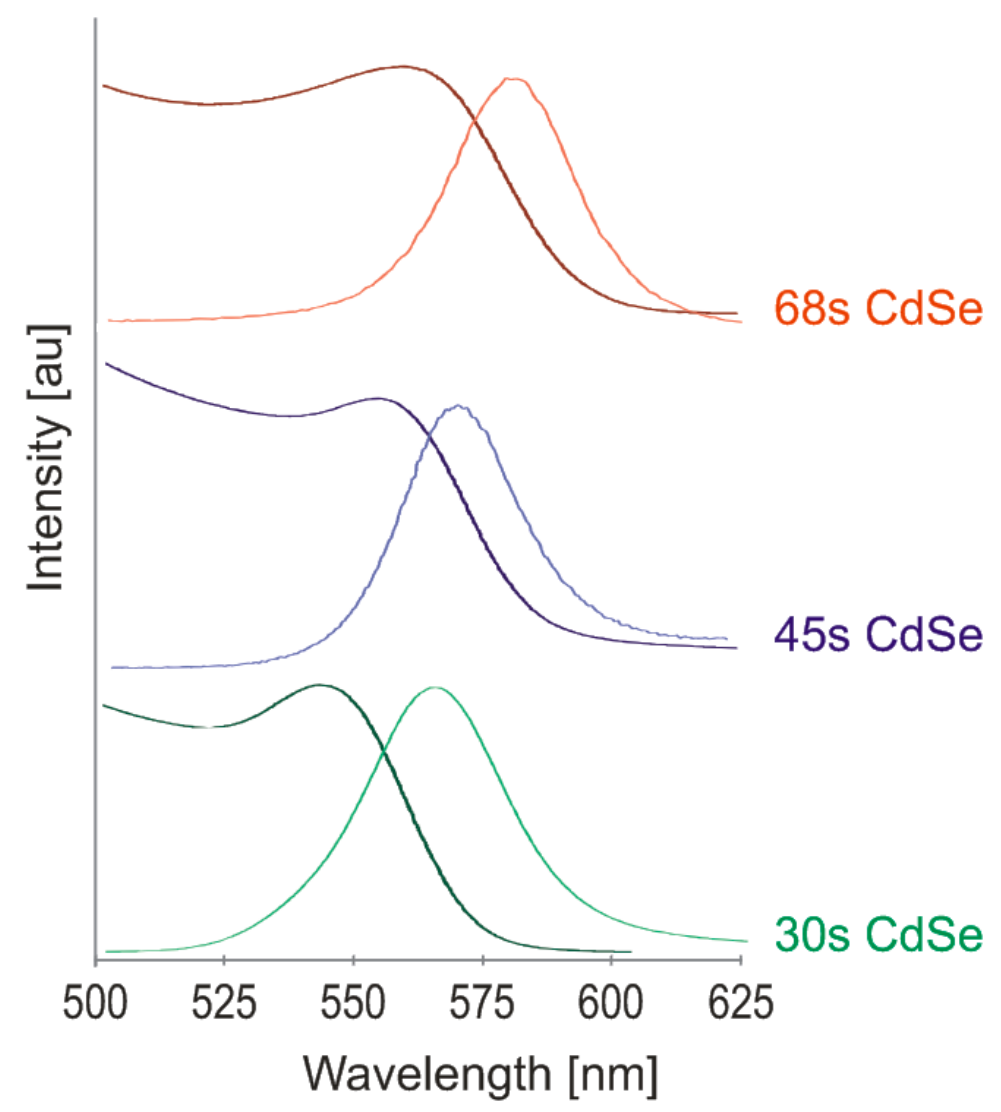

Figure 2-2. Absorbance (left) and PL spectra (right) of CdSe QDs in chloroform solution synthesized with increasing growth times. (Spectra are vertically shifted for clarity.)

The reproducibility of this synthetic approach was studied by independently reproducing the synthesis in triplicate using identically prepared solutions and allowing each to react for the 
same amount of time. In these tests, the full reaction mixture was quenched by rapidly adding room temperature ODE to double the reaction volume. These three syntheses were purified separately and resuspended in chloroform. The samples were diluted and normalized by their absorption at the excitation wavelength for spectra comparisons The spectra for the three separate syntheses indicate high reproducibility of the process, as they are nearly identical with an overall emission peak deviation of $\pm 3.0 \mathrm{~nm}$ (Figure 2-3). This corresponds to a size variation of $\sim 0.07 \mathrm{~nm}$ implying single monolayer control of CdSe core growth. ${ }^{75}$

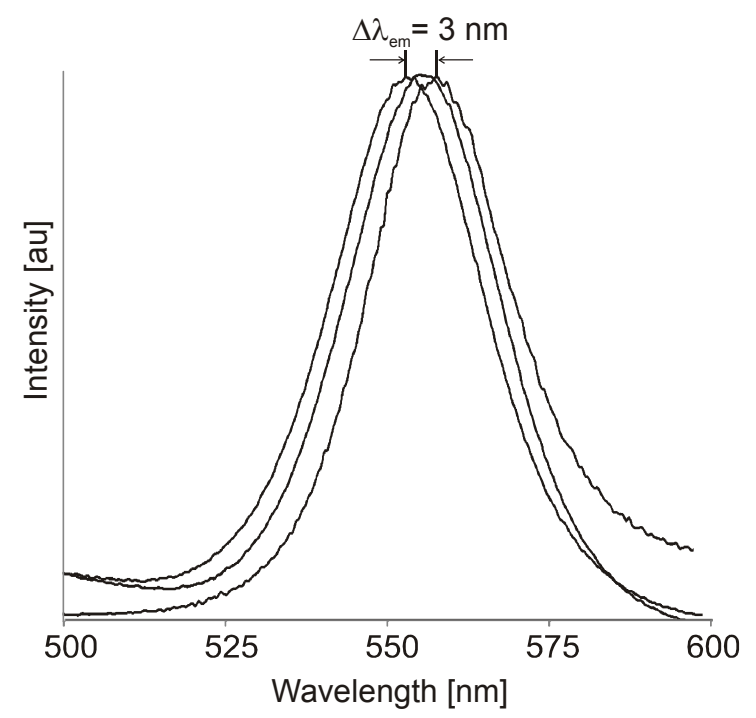

Figure 2-3. Fluorescence spectra of three distinct CdSe core reactions quenched after identical growth time. $\Delta \lambda=3 \mathrm{~nm}$ across all three solutions.

\subsubsection{ZnS shell growth and characterization}

Previous syntheses of ZnSe and PbS QDs suggested a possible route to $\mathrm{ZnS}$ using a simple, non-pyrophoric approach. ${ }^{45,76}$ Using zinc stearate and TOP(S) as reactant sources avoids the use of air-sensitive dimethyl zinc and bis-trimethylsilyl sulfide, both of which are significant combustion hazards. 
For ZnS shell growth, CdSe cores were typically purified by precipitation with methanol and resuspended in chloroform before use; however, initial studies indicate that this is unnecessary as long as low-boiling point solvents and impurities are removed by vacuum. The slow injection of the zinc and sulfide precursors allowed time for growth of $\mathrm{ZnS}$ materials on the CdSe core. The slow delivery of reagents combined with the low temperature should reduce opportunities for the formation of $\mathrm{ZnS}$ particles. The presence of long chain and/or amphiphilic surfactants and solvents, many of which are solids at room temperature required the addition of an additional solvent to prevent solidification of the product mixture. Purification of the core-shell QDs from unreacted stearate, TOP, and HDA was carried out by methanol precipitation and centrifugation. CdSe/ZnS core/shell QDs were dried and dissolved in chloroform for characterization.

The thickness of the ZnS shell on the CdSe core was controlled by the amount of zinc and sulfur precursors. The amount of precursors added was determined using a single ZnS (wurtzite) monolayer of $3.6 \AA$ as basis for calculating the molar amounts of zinc and sulfur to be added to the exterior of the CdSe QDs based on molar concentration of the QDs and the estimated diameter of the QDs. For example, CdSe core diameter can be determined to be 3.0 $\mathrm{nm}$ from TEM data. The spherical volume of the core is then calculated using by $\frac{4}{8} \pi r^{8}$ to be 14.1 $\mathrm{nm}^{3}$. If 2 full monolayers of $\mathrm{ZnS}$ are desired on the CdSe QD core, this equates to a shell thickness of $0.72 \mathrm{~nm}$ based on the wurtzite structure. Adding the shell thickness to the core radius gives a radius of the full core/shell QD to be $2.22 \mathrm{~nm}$. The spherical volume of the total core/shell QD is $45.8 \mathrm{~nm}^{3}$. Subtracting the volume of the core only from the volume of the core/shell QD gives the volume of the shell only, which, in this example, is $31.7 \mathrm{~nm}^{3}$. From the volume of $1 \mathrm{ZnS}$ unit, in can be estimated that this volume of $\mathrm{ZnS}$ shell should contain 
approximately $270 \mathrm{ZnS}$ units or $810 \mathrm{Zn}$ and $\mathrm{S}$ atoms. From this information, the molar equivalents of material are calculated. A typical reaction contains a ten-fold excess of material.

Energy-dispersive x-ray spectroscopy (EDS) measurements confirmed the presence of cadmium and selenium core materials and zinc and sulfur shell materials by their characteristic x-ray emission (Figure 2-4). Dissolved QDs were deposited on clean highly ordered pyrolytic graphite (HOPG), which accounts for the high carbon peak along with a small contribution from the long-chain aliphatic surfactant molecule. Phosphorous peaks indicate the presence of organic TOPO molecules. The chlorine peak is likely due to the presence of residual chlorinated solvents or chlorinated contaminants from solvent or reaction mixture. No effort was made to quantify the chemical composition from the EDS spectrum, however, EDS unambiguously confirms the elemental components of the material to be as expected. More information about the location of each material within the QD and binding state was determined by alternative methods.

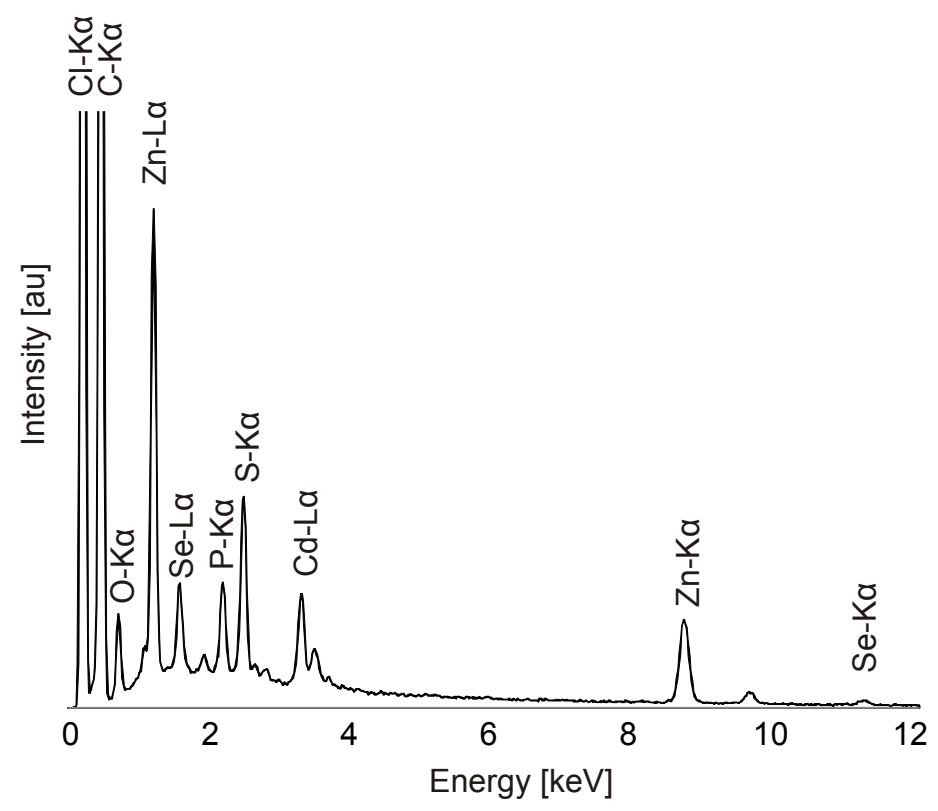

Figure 2-4. Energy dispersive x-ray spectroscopy of CdSe/ZnS core/shell QDs verifying the qualitative elemental composition. 
Emission spectra from CdSe-TOP QDs were compared to the same CdSe QDs after the addition of a ZnS overlayer (Figure 2-5). Solutions with similar optical density at the excitation wavelength of $480 \mathrm{~nm}$ were compared. Each spectrum was collected with solutions of QDs at an optical density less than 0.1 to minimize reabsorption. As expected from the larger bandgap of the overcoating $\mathrm{ZnS}$ and as observed by previous authors, ${ }^{77}$ there is a slight $(\sim 3 \mathrm{~nm})$ red shift of the emission from overcoated QDs, but the ZnS overcoat does not significantly impact the FWHM of the emission peak. The photoluminescence intensity of the ZnS coated QDs is about 1.5 times greater than that of the native CdSe QDs. This quantum yield enhancement has been observed by previous authors to be $15-50 \%$ depending on the shell thickness and is believed to arise due to capping of quenching surface states from disordered CdSe and CdO impurities on the as-synthesized CdSe. ${ }^{64}$

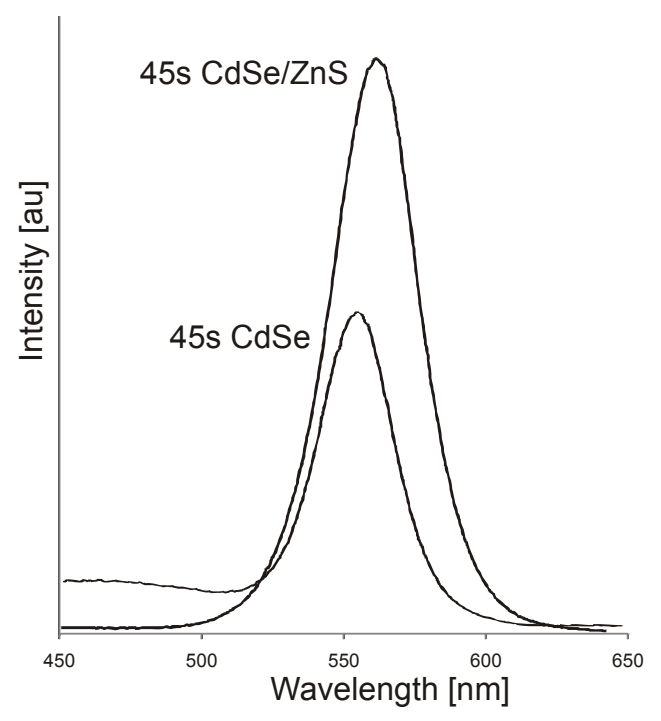

Figure 2-5. PL spectra of CdSe cores grown for 45 seconds, purified, and redispersed in chloroform and the same CdSe cores after ZnS shell addition ( 4 monolayers) and purification.

The PL quantum yield ${ }^{43}$ was determined to quantify the quality of QD excitation and emission. PL spectra of the samples before and after shell addition were compared with the spectrum of an organic dye of known quantum yield with a similar peak emission wavelength. 
All samples were prepared with similar optical densities at the excitation wavelength. By comparing the integrations of the PL spectra from the QD sample and the organic dye, the PL quantum yield of the CdSe cores was determined to be approximately $30 \%$. Upon addition of the $\mathrm{ZnS}$ shell, quantum yield increased to $78 \%$.

The size of the CdSe cores was characterized directly and indirectly by several methods. The UV-Vis data were analyzed in the method of Brus, et al using the effective mass model equation which provides a direct correlation between UV-Vis peak and particle size. ${ }^{50,78}$ This analysis provided size and concentration estimates for the CdSe cores synthesized for different periods of time. CdSe QD core diameters were also estimated using Yu's previously published study of absorbance and size correlation based on empirical data from multiple authors. ${ }^{49}$ These estimates are provided for comparison (Table 2-1). Size and monodispersity were confirmed by TEM (Figure 2-6). TEM images obtained of the CdSe QDs before and after ZnS coating show similar uniformity and a slight size increase. Particle size analysis of the TEM images yielded CdSe core diameters of $2.91 \pm 0.05 \mathrm{~nm}(\mathrm{n}=122)$ and CdSe/ZnS diameters of $5.43 \pm 0.08 \mathrm{~nm}(\mathrm{n}$ = 278). Size was determined by applying a Gaussian fit to a size distribution histogram generated from measurements taken using Image J.

Table 2-1. Comparison of size determinations for three QD CdSe cores synthesized with increasing growth times based on experimental data fit, effective mass model calculation, and TEM observations.

\begin{tabular}{lllllll}
\hline $\begin{array}{l}\text { Reaction } \\
\text { time }[\mathrm{sec}]\end{array}$ & $\begin{array}{l}\lambda \text {-Absorbance } \\
{[\mathrm{nm}]}\end{array}$ & $\begin{array}{l}\lambda \text {-Emission } \\
{[\mathrm{nm}]}\end{array}$ & $\varepsilon\left[\mathrm{cm}^{-1} \mathrm{M}^{-1}\right]$ & $\begin{array}{l}\text { Diameter: } \\
\text { Yu [nm] }\end{array}$ & $\begin{array}{l}\text { Diameter: } \\
\text { Eff. Mass [nm] }\end{array}$ & $\begin{array}{l}\text { Diameter: } \\
\text { TEM [nm] }\end{array}$ \\
\hline 30 & 541.0 & 546.5 & 92,031 & 2.87 & 2.86 & 2.78 \\
45 & 545.0 & 552.5 & 92,818 & 2.94 & 2.91 & 2.91 \\
68 & 557.0 & 566.0 & 115,576 & 3.19 & 3.05 & 3.05 \\
\hline
\end{tabular}



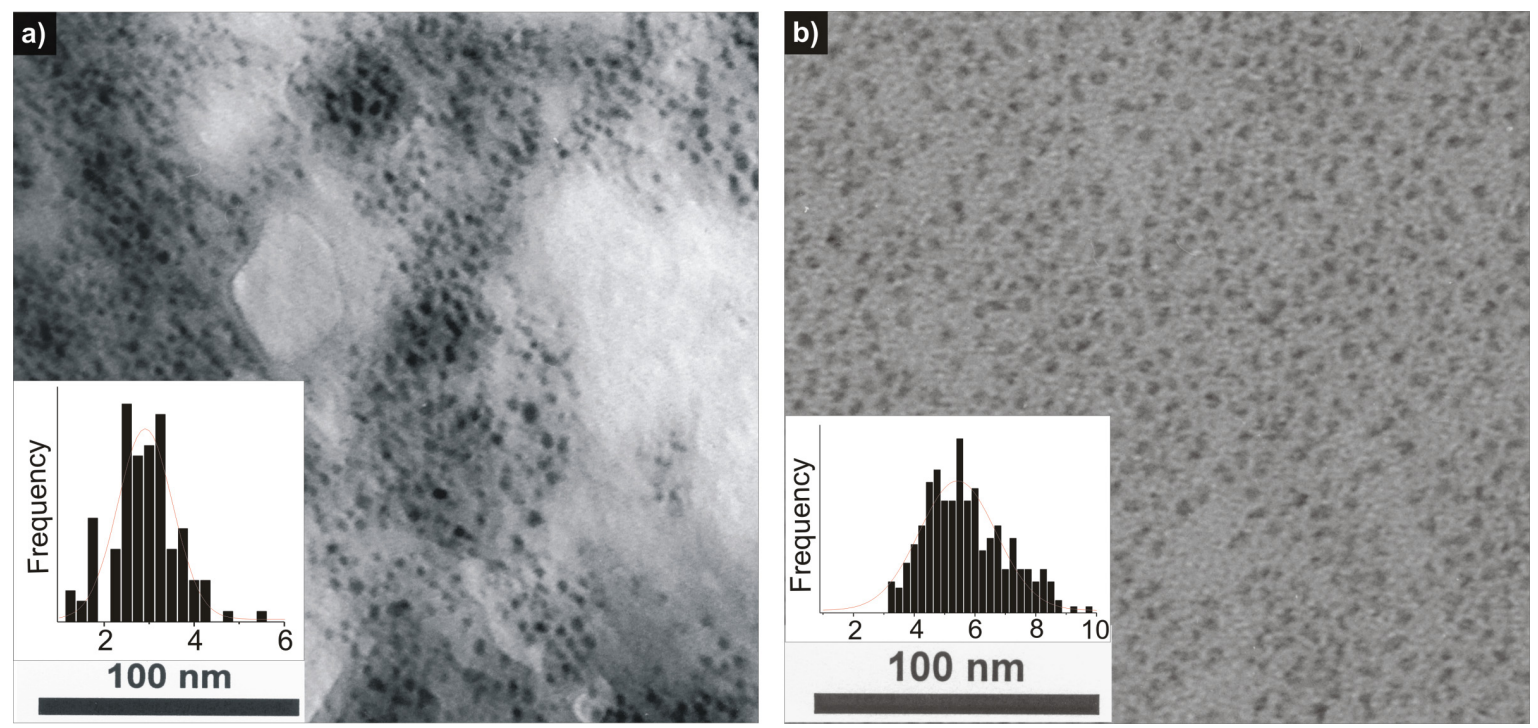

Figure 2-6. TEM of (a) CdSe cores synthesized for $45 \mathrm{sec}$ and (b) of same CdSe cores with $\sim 4$ monolayers of ZnS. Insets show histogram and Gaussian fit of particle size.

X-ray photoelectron spectroscopy (XPS) data further confirms the qualitative and quantitative composition of the core and shell materials. The high-resolution spectrum (Figure 2-7) shows the expected peaks in the $C d\left(3 d_{5 / 2}, 3 d_{3 / 2}\right)$, Se $(3 d), Z n\left(2 p_{3 / 2}\right)$, and $S(2 p)$ regions. No overt signs of oxidation were observed after being exposed to light and air for several weeks. Formation of $\mathrm{SeO}_{2}$ would have been observable by a shift in the selenium peak had oxidation occurred. This result indicates that the ZnS shell sufficiently protects the CdSe core from oxidation and degradation. 


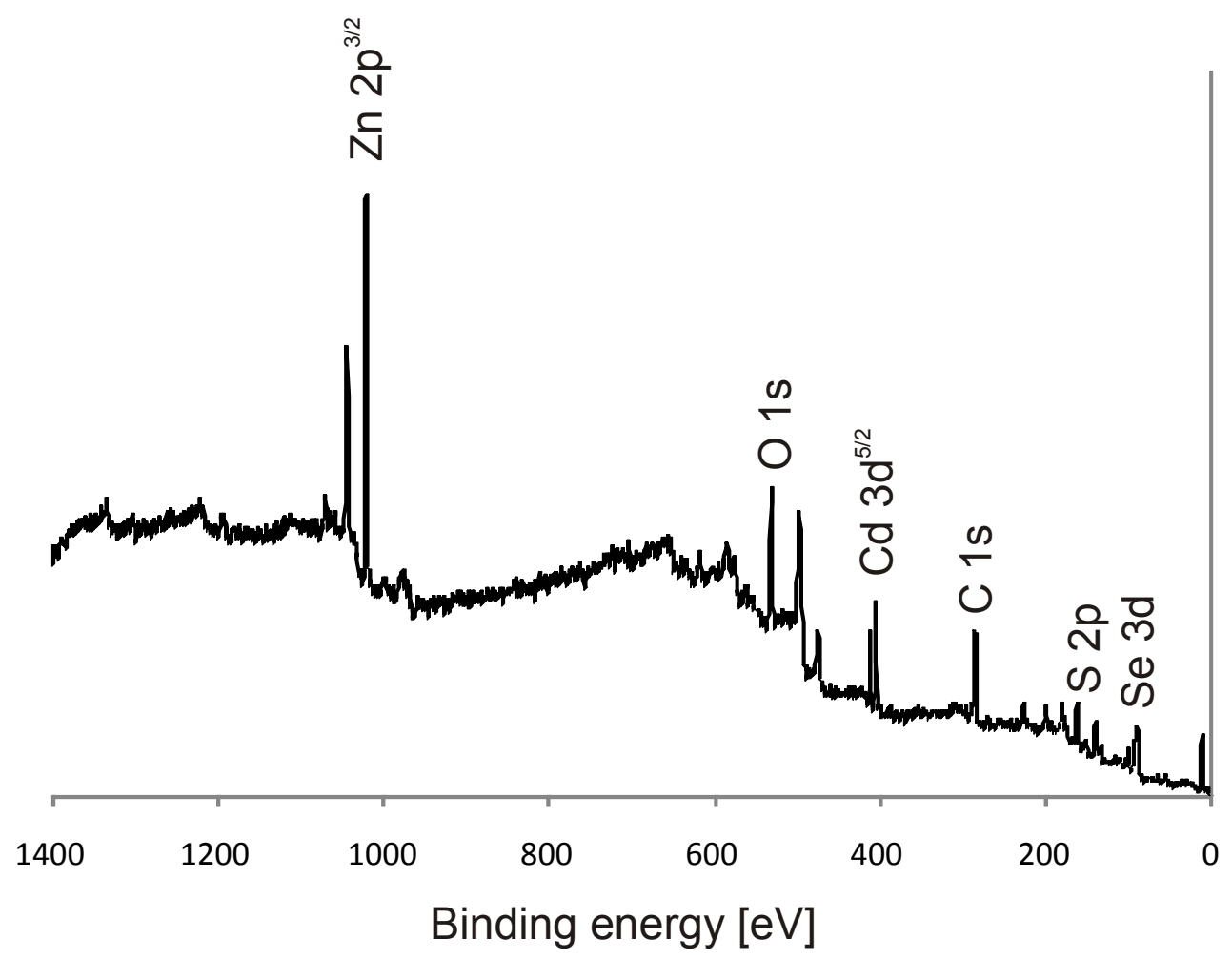

Figure 2-7. XPS spectrum of CdSe/ZnS QDs.

\subsection{Conclusion}

The synthetic method described above is simple and inherently safer than previously published methods, requiring less specialized equipment and tolerating greater flexibility in reaction conditions. Absorbance and emission spectra indicate sharp peaks and low size distribution for CdSe cores, a slight red-shift with addition of ZnS shell due to increase in diameter and significant PL QY enhancement for core-shell QDs because surface vacancies are passivated by the $\mathrm{ZnS}$ coat.

The QDs synthesized by this approach show characteristics similar to those produced through more technically challenging syntheses. Primarily, the emission peak of the QDs is tunable through control of reaction time as evidenced by a triplicate reaction at 45 seconds to 
produce QDs of $2.9 \mathrm{~nm}$ diameter and an emission peak wavelength variation of $\pm 3.0 \mathrm{~nm}$ and a size variation of $\sim 0.07 \mathrm{~nm}$. Size and emission wavelength control along with the ability to stabilize and reduce degradation of CdSe QDs through the addition of a ZnS shell dramatically increases the relative intensity of the emission peak with a slight red-shift as expected. 


\section{Chapter 3. QD surface modification and functionalization}

\subsection{Introduction}

QDs have applications in a variety of areas, but one of the most prolific is in biomolecular conjugation and imaging. Fluorescent labels have enabled many researchers to track physical and chemical interactions of proteins, cells, and other biomolecules. ${ }^{73,79,80}$ Organic fluorescent dyes have also been used for such studies, however, QDs offer several advantages over fluorescent dyes (Table 3-1). ${ }^{78}$

Table 3-1. Comparison of QDs and organic fluorescent dyes for biological labeling experiments.

\begin{tabular}{ll}
\hline \multicolumn{1}{c}{ QDs } & \multicolumn{1}{c}{ Dyes } \\
\hline Single source excitation for many QDs & One excitation source per dye \\
Narrow emission spectra & Emission spectra subject to broadening \\
Bright fluorescence & Limited brightness \\
High photostability & Fast photobleaching \\
Emission largely insensitive to chemistry & Functional groups can modify emission \\
\hline
\end{tabular}

A typical QD synthesis results in a core/shell QD with long-chain organic surfactant molecules attached. These QDs are soluble in several organic solvents such as chloroform, toluene, and hexane. In these solvents, the TOP molecules are bound to the surface selenide or sulfide atoms. Additional TOP-O is also present and contributes $\sim 70 \%$ to surface coverage. ${ }^{28}$ However, when the QDs are dissolved in pyridine, the TOP-O is displaced by the pyridine leading to decreased surface coverage. ${ }^{35}$ This results in a greater chance of aggregation in solution, but also makes the nanoparticles easier to dry and adhere to surfaces. Other long 
chain alkanes can also be attached to the surface of the QD through ligand exchange imparting various degrees of hydrophobicity to the particles.

In order to utilize organic synthesized QDs in biological and other aqueous media, hydrophilic surfactants must be attached. Several small molecules have been successfully attached to semiconductor nanoparticle substrates using terminal acid and amine groups such as mercaptopropionic acid (MPA) and aminoethane thiol (AET). Acid and amine terminated molecules are desirable because they can be easily modified to attach to peptides such as antigens, antibodies, or enzymes through readily available chemistry. Scheme 3-1 conceptually illustrates 1-ethyl-3-[3-dimethylaminopropyl] carbodiimide hydrochloride (EDC) and N-hydroxysulfosuccidimide (NHS) chemistry typically used for peptide coupling. This method can also be used to bind QDs to biomolecules through the activation of carboxylate terminated QDs with amine terminated proteins. Binding targets can also be interchanged so that amine terminated QDs are bound to carboxylate terminated biomolecule.

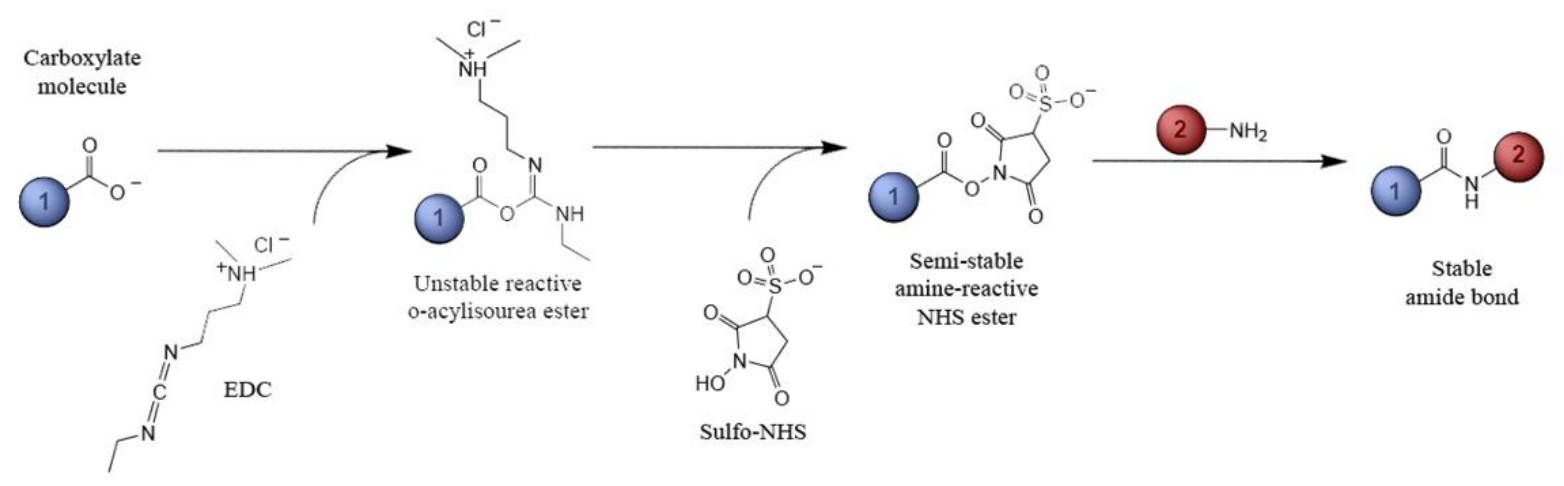

Scheme 3-1. A generalized scheme of carbodiimide (EDC) and hydroxy succinimide (NHS) coupling of carboxylate-terminated molecules (1) with amine-terminated molecules (2).

To achieve displacement of existing surfactant molecules for the desired molecules, ligand exchange is performed ${ }^{81}$ For most exchanges, simply mixing the original and desired 
ligands with gentle mixing is sufficient to remove the original ligand and adsorb a new ligand on the particle surface. Length of time to achieve complete removal and coverage varies depending on molecular weight and orientation of each species. As ligand exchange proceeds, the particles flocculate out of one phase into the other.

A similar surface modification process was used to produce amphiphilic particles. Assynthesized QDs were encapsulated in a copolymer. Polymer synthesis and exchange was performed similar to previously published procedures. ${ }^{82,83}$ Polymers have also been explored as nanoparticle surface modifiers. ${ }^{84}$ Polymer encapsulated particles offer a new range of variability in chain length and amphiphilic properties for solubility and self-assembly of nanoparticles.

\subsection{Experimental}

QDs synthesized as described in Chapter 2 were purified and modified with hydrophobic, hydrophilic, and amphiphilic surfactants. Analysis and characterization of these modifications are presented.

\subsubsection{Surface modification procedure}

We considered two approaches to modify the surface of the QDs and to provide coupling points for biomolecular analytes. Ligand exchange of the TOPO stabilizing ligands was performed at room temperature using an excess of MPA (Scheme 3-2). $20 \mu \mathrm{l}$ of MPA was added to $250 \mu \mathrm{l}$ of $\mathrm{CdSe} / \mathrm{ZnS} \mathrm{QDs}$ at $\sim 50 \mu \mathrm{M}$ in chloroform. The solution was left to react for $\sim 2$ hours after which obvious precipitation of QDs was observed. The nanoparticles were washed three times by centrifuging at 1000 xG for 2 minutes, removing supernatant solvent, and redispersing in $200 \mu \mathrm{l}$ of chloroform. Finally, $200 \mu \mathrm{l}$ of $0.1 \mathrm{M}$ PBS buffer at pH 7.4 was added. $\mathrm{NaOH}$ was then added dropwise until the QDs were resuspended in solution (typically $\mathrm{pH} \sim 10$ ). In this matrix, the QDs formed a stable suspension for $\sim 5$ days. 


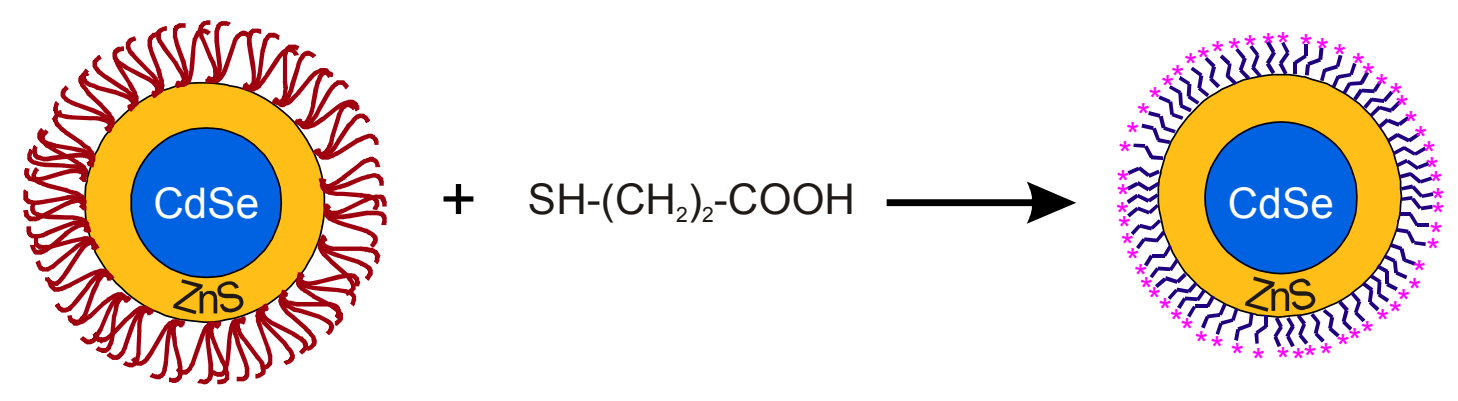

Scheme 3-2. Conceptual schematic of hydrophobic-to-hydrophilic ligand exchange.

An alternative method for altering the hydrophobicity of QDs is polymeric encapsulation (Scheme 3-3). A polyacrylic acid (PAA) backbone was modified with $\sim 80 \%$ octylamine (OA) chains following the method of Shen, et al. ${ }^{85} 2.5 \mathrm{~g}$ polyacrylic acid was dissolved in $35 \mathrm{ml}$ DMF. EDC was added to activate the carboxylic acid groups. $3.2 \mathrm{ml}$ octylamine was added and formed amide bonds to the activated acid groups on the polyacrylic backbone. After stirring overnight, excess solvent was removed and $10 \mathrm{ml}$ distilled water was added to precipitate the copolymer. The polymer was isolated by centrifugation and redissolved in $20 \mathrm{ml} 1 \mathrm{M} \mathrm{NaOH}$. To encapsulate QDs, $1 \mathrm{~g}$ of polymer was added to $100 \mathrm{mg}$ QDs in $\mathrm{CHCl}_{3}$. The mixture was sonicated until the polymer was completely dissolved. The solution was filtered through $0.1 \mu \mathrm{m}$ Anodisc filter and adjusted to $\mathrm{pH}$ 6. QDs prepared this way were stable for over 1 month.

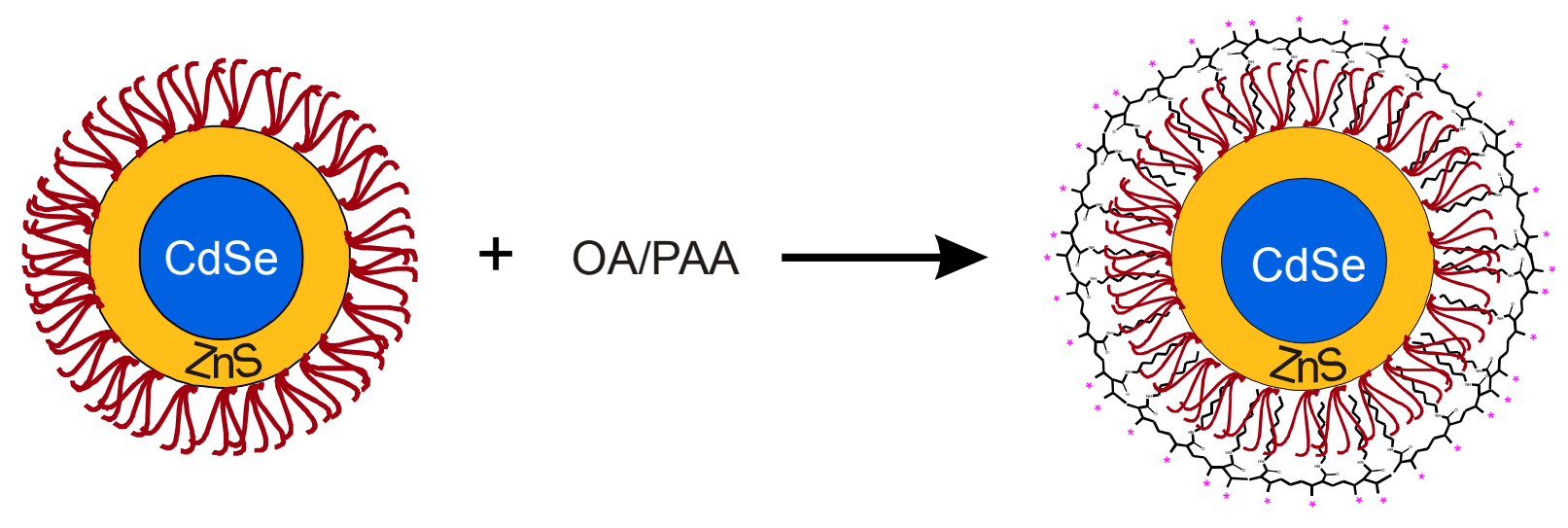

Scheme 3-3. Conceptual schematic of polymeric encapsulation of QD. 


\subsubsection{Protein binding}

To confirm protein-binding specificity, antibody labeled QDs were assayed against complementary antigens on engineered substrates and cellular substrates. Following organic or polymeric ligand modification of the QDs, the particles were conjugated to bovine serum albumin (BSA) antibody via acid activation of the QD ligand using EDC and NHS to bind to amine groups on the antibody. BSA antigen labeled with fluorescein isothiocyanate (FITC) was immobilized on glass substrates by microcontact printing. ${ }^{86}$ This procedure generates periodic patterns of BSA and bare substrate areas (Figure 3-1). Antibody conjugated QDs were then reacted with the stamped region and rinsed thoroughly. Confocal microscopy was used to confirm selective binding of the QDs only to BSA regions by colocalization of the FITC (515 nm) and QD (590 nm) emissions.

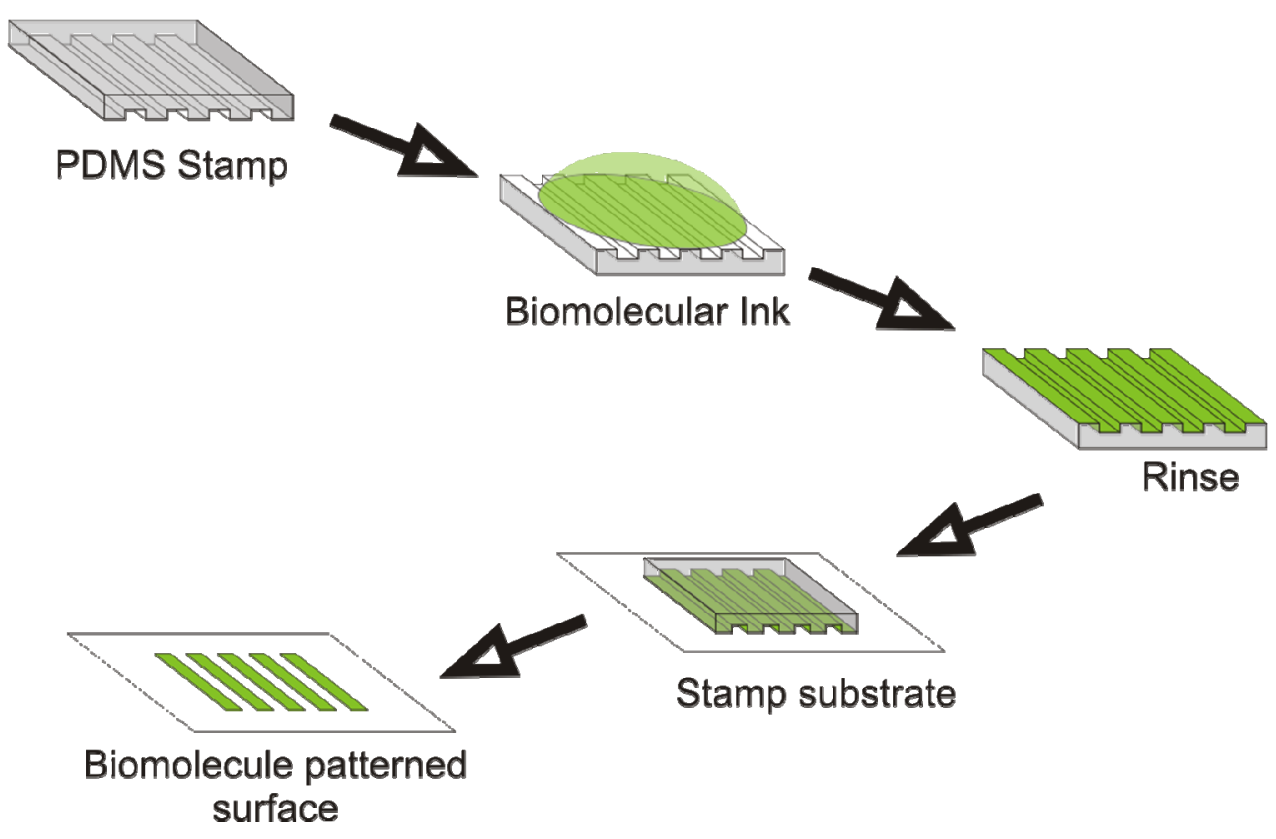

Figure 3-1. Microcontact printing procedure 
Atomic force microscopy (AFM) in fluid was also performed on the same substrates described above. Height traces of the engineered substrates were compared before and after conjugation with antibody labeled QDs.

\subsubsection{Cell studies}

Cellular assays were performed on a human lymph node endothelial cell line. SVEC-410 cells were cultured in Dulbecco's Minimal Eagles Medium (DMEM) supplemented with 1\% fetal bovine serum and $1 \%$ penicillin and streptomycin in $5 \% \mathrm{CO}_{2}$ humidified atmosphere at $37^{\circ} \mathrm{C}$. These cells express $\beta$-catenin, a surface membrane protein, which plays a role in embryogenesis and has been linked to cell growth in cancerous tumors including basal cell carcinoma, colon, and breast cancers. ${ }^{87}$ Secondary labeling of the protein was performed with rabbit IgG. Cells were fixed for imaging with confocal and fluorescent microscopy.

\subsection{Results and discussion}

\subsubsection{Surface modification characterization}

ATR-FTIR spectra of the reactant mixture before and after purification confirm removal of unbound surfactant and excess reaction solvent. Assuming nearly complete removal of unbound organic molecules, TOPO will be the primary ligand with an IR signature. The spectrum of neat TOPO (Figure 3-2a) shows characteristic peaks for C-H stretching from 2950 $\mathrm{cm}^{-1}$ to $2860 \mathrm{~cm}^{-1}$, the $\mathrm{CH}_{3}$ bending region at $1465 \mathrm{~cm}^{-1}$, and the $\mathrm{P}-\mathrm{O}$ stretching region near $1145 \mathrm{~cm}^{-1}$. The IR spectrum of the QDs (Figure 3-2b) shows $\mathrm{C}-\mathrm{H}$ bending and stretching regions attributable to TOPO. However, the $\mathrm{P}-\mathrm{O}$ stretching region is absent, and only the $\mathrm{P}-$ $\mathrm{CH}_{3}$ deformation vibration is observed at $910 \mathrm{~cm}^{-1}$. 


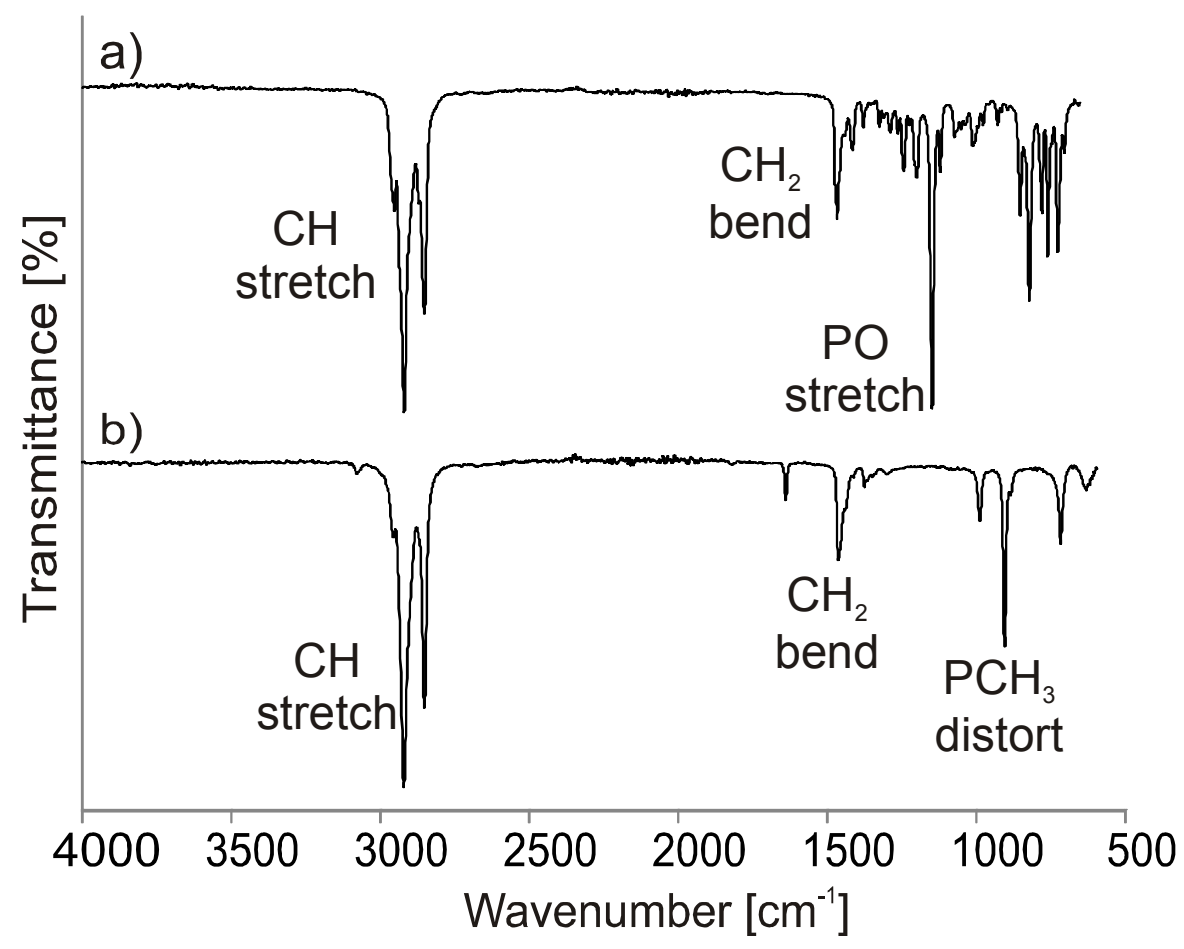

Figure 3-2. Infrared spectra of (a) neat TOPO and (b) TOP surfacted QDs.

Ligand exchange of TOPO for mercaptopropionic acid (MPA) was performed at room temperature in ambient conditions and could be visually confirmed by QDs precipitating out of the organic solvent phase and dispersing in aqueous phase solution (Figure 3-3 photo insets). IR spectroscopy was used to track the process of ligand exchange over 24 hours. After 12 hours, no further change could be observed in the spectrum indicating complete exchange. The spectrum of the final hydrophilic product after purification by methanol precipitation to remove excess reactants and solvents (Figure 3-3) shows the disappearance of the aliphatic stretching and bending frequencies at $2950-2850 \mathrm{~cm}^{-1}$ and $1425 \mathrm{~cm}^{-1}$ consistent with the removal of TOPO and the appearance of the $\mathrm{OH}$ stretching region at $3350 \mathrm{~cm}^{-1}$; the $\mathrm{C}=\mathrm{O}$ stretching region at $1650 \mathrm{~cm}^{-1}$ confirms the presence of MPA. 


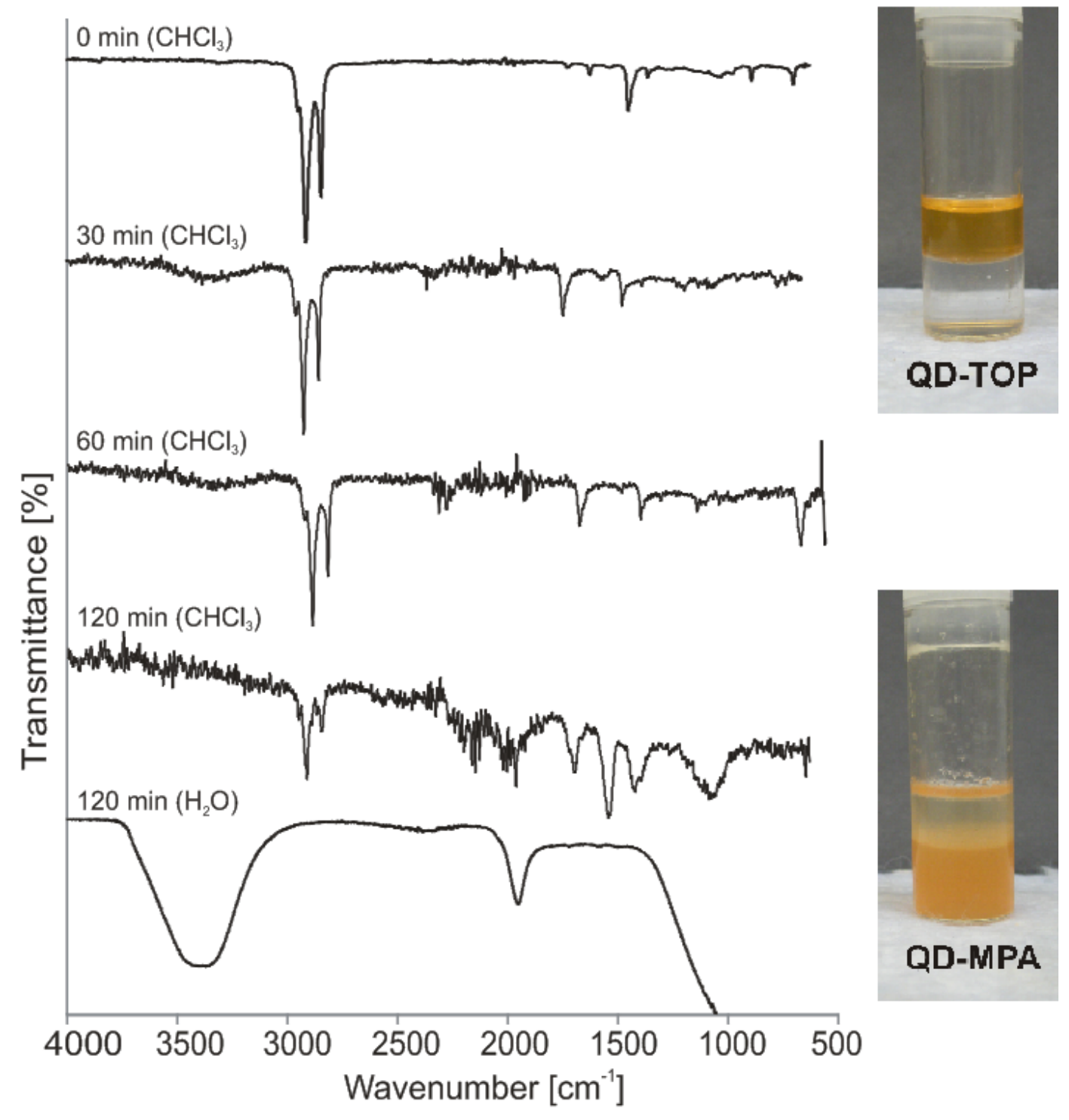

Figure 3-3. Time series of IR spectra of ligand exchange of TOP for MPA.

\subsubsection{Protein binding studies}

Antibody conjugated QDs were reacted with engineered protein substrates. Confocal microscopy was used to confirm selective binding of the QDs only to BSA regions by colocalization of the FITC $(515 \mathrm{~nm})$ and QD $(590 \mathrm{~nm})$ emissions. The red fluorescent QDs and green fluorescent FITC, when colocalized, appear yellow (Figure 3-4). 

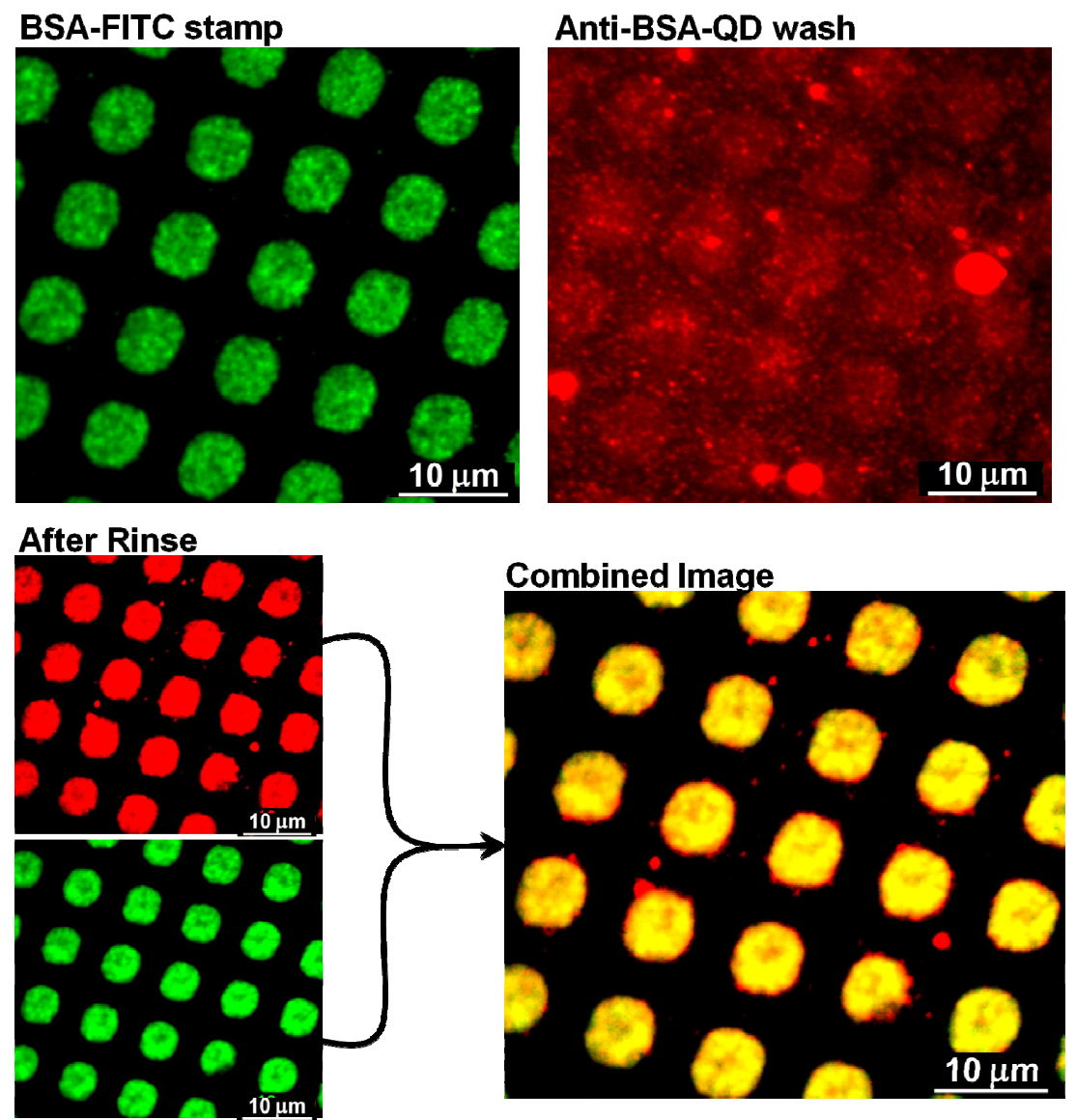

Figure 3-4. Confocal microscopy images of QD emission (DAPI filter; red-channel) and FITC emission (FITC filter; green-channel).

Liquid phase AFM offers unique insight on the bioconjugation of QDs with proteins. When coupled with confocal microscopy, substrates can be unambiguously studied. Future work will use this setup to study binding efficiency of various antigen/antibody couplings as a function of QD surfactant.

Atomic force microscopy (AFM) in fluid was also performed on the same samples (Figure 3-5). The presence of antigen stamped patterns are readily visible height trace of the BSA stamp only (green). Fluid AFM studies indicate that the height increases from 2-4 nm for BSA stamped regions alone to 10-15 nm after conjugation with Anti-BSA labeled QDs (red line). 

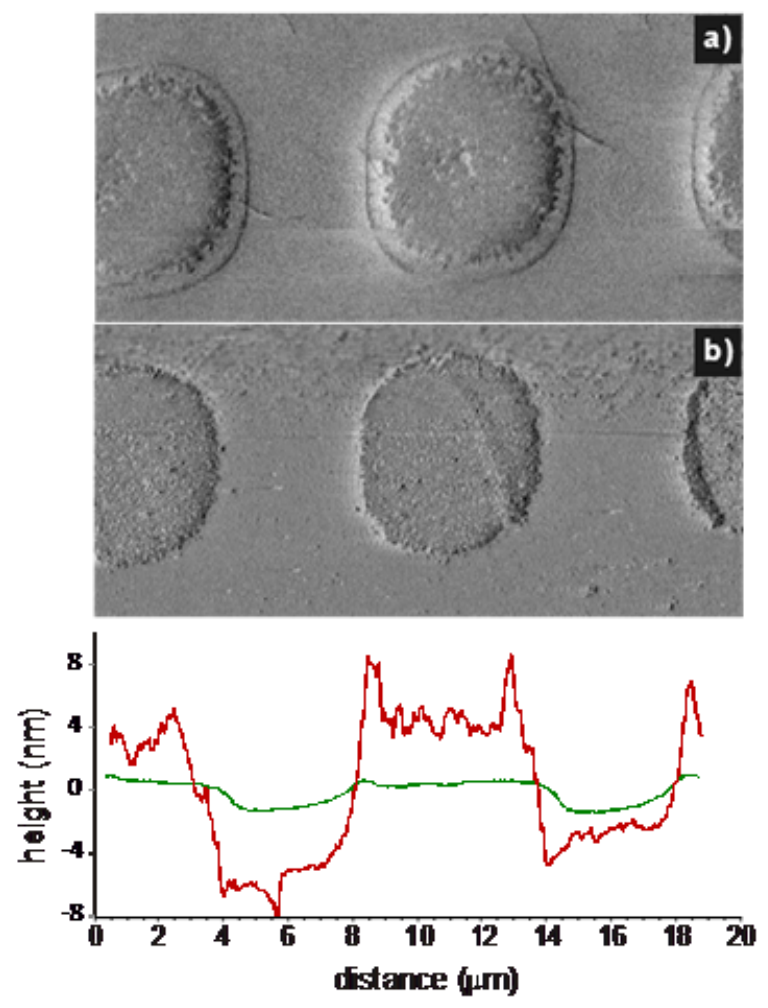

Figure 3-5. Fluid AFM images of (a) BSA stamp and (b) BSA stamp with MPA-QDs. Height traces are of BSA stamp before (green) and after (red) QD conjugation.

Cellular studies were performed on a human endothelial cell line. Specific targeting of QDs was achieved by labeling the QD with anti- $\beta$-catenin and incubating the cell cultures with Ab-QDs (red fluorescing). The cells were permeabilized and incubated with QDs labeled with anti- $\beta$-catenin to demonstrate specific targeting. Secondary labeling with FITC-lgG (green fluorescing) confirms that the QDs are bound to the proteins rather than non-specific adsorption to other cellular structures (Figure 3-6). 

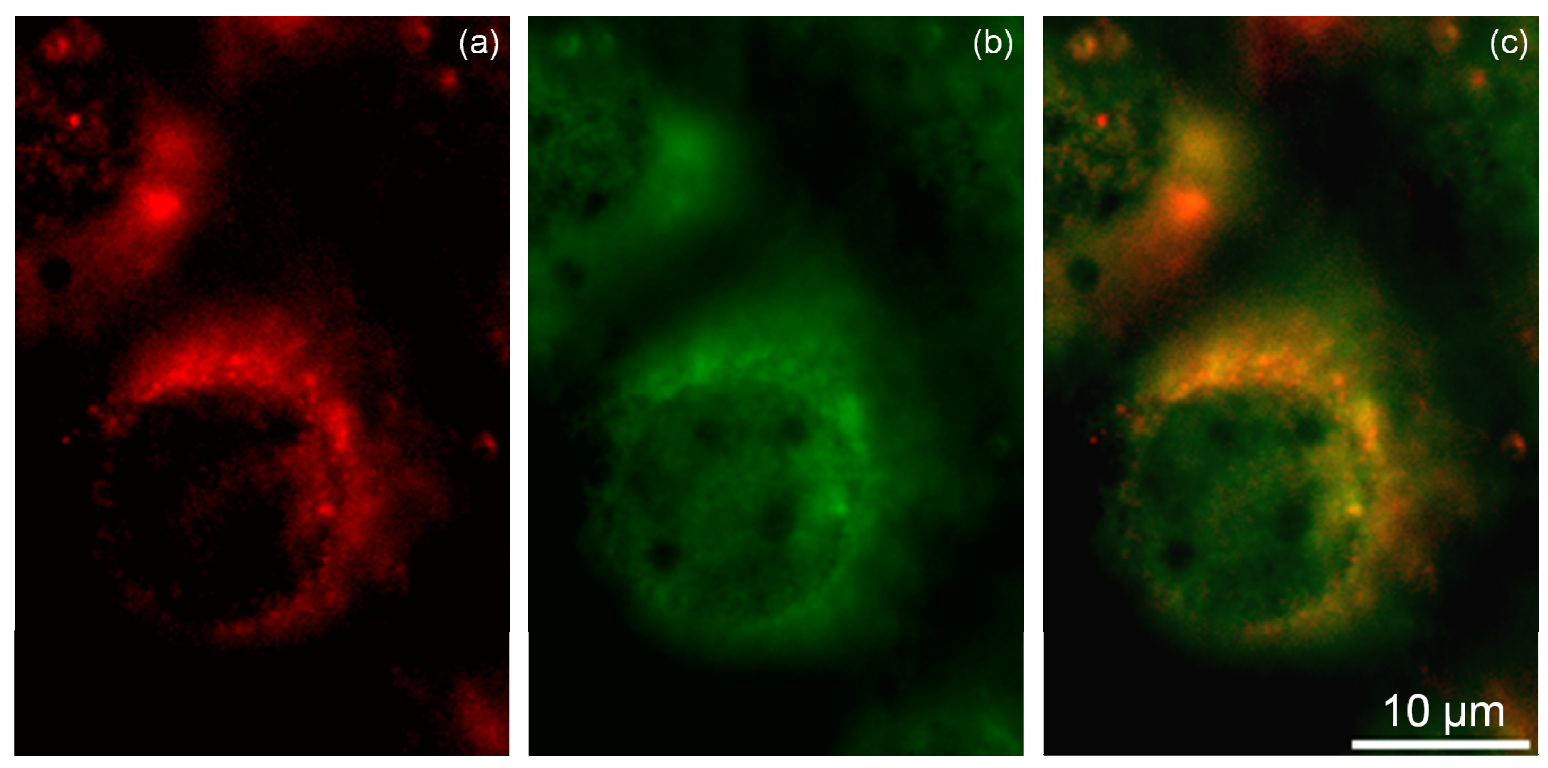

Figure 3-6. Confocal image of cells after conjugation with Ab-QDs and immunolabeling. a) red channel showing $600 \mathrm{~nm}$ QDs, b) green channel showing FITC (514nm), and c) colocalization of red and green produce yellow.

\subsection{Conclusion}

Surface modification presents an easy method to control binding, optical, and physical properties of QDs. Surface molecules can be easily displaced by appropriate solvent or other molecules through ligand exchange. The process of ligand exchange can also be used for purification and isolation of desired products in high purity and yield.

Protein and cellular studies demonstrate that the QDs synthesized by the method outlined in Chapter 2 have similar reactivity to other QDs. Further, surface modification of the QDs does not interfere with in vitro antibody-antigen interactions. Success of this research in demonstrating specific binding of antibody conjugated QDs to targeted antigens on cellular substrates is a significant step towards cancer cell labeling. Future work could include use of other known cancer cell markers, development of cancer marker screening devices, facilitate 
tracking of cancer cell migration, and provide a vehicle for delivery of targeted therapeutic treatments.

While QDs continue to be exploited for their unique fluorescent properties in many areas, biological conjugation is complex because of the native QD surfactants formed during synthesis. Ligand exchange and polymeric encapsulation have proved to be two important and successful methods for modifying the surface and reactivity of QDs. 


\section{Chapter 4. CdS Nanorods}

\subsection{Introduction}

Nanoscale constructs have potential in many applications because many properties can be adjusted and tuned to fulfill specific functions. Nanorods photoluminesce, which is the hallmark of quantum confined nanostructures, but they can also produce linearly polarized emission. ${ }^{88}$ Nanorods are in development as solar cells, light-emitting diodes, photodetectors, lasers, and quantum computers. ${ }^{89,90}$ The properties of nanorods are controlled by material, dimensions, shape, and crystal phase. The most common materials are metals and semiconductors. Of the semiconductors, II-VI materials have received the most attention because of the pioneering work done on QDs of CdS, CdSe, CdTe, and others. ${ }^{57,91}$

Nanorods can be defined by their aspect ratio - that is, the ratio of their length to width (diameter). A zero-dimensional nanoparticle would have an aspect ratio of 1 , whereas a very long, very thin one-dimensional structure can have an aspect ratio $>1000$. Although the terms have been used interchangeably in literature, typically the term nanorod describes a structure with an aspect ratio greater than 1 but less than 100, while nanowire describes structures with aspect ratios greater than 100 . There are many morphological considerations for semiconductor nanorods. A unique set of physical, chemical, and electronic properties of nanorods can be defined based on the crystal phase and orientation of the material as well as if the structure is multi-crystalline or a single crystal. Material strength is greatly affected by crystal phase and orientation. Rods with multiple crystal phases tend to form multi-rod structures such as tripods, tetrapods, or more complex structures such as nano trees, arrows, or pyramids. Aspect ratio and size are perhaps the most recognizable physical property of nanorods. 
The most common crystal structures for II-VI semiconductor materials are zinc blende, wurtzite, or rock salt. The crystallinity and orientation of these materials can produce anisotropic growth along one or more planes. Initial research on anisotropic growth of semiconductor nanorods heavily borrowed from CdSe QD syntheses and used a mixed surfactant approach to retard growth of one crystal plane. ${ }^{39,58}$

A simple, readily available method of controlling size and shape of single rod species is with a templated approach. Analogous to macroscale molding techniques, a template with the inverse of the desired morphology is created and the semiconductor material is then deposited or grown inside of the mold. Templates made from aluminum oxide membranes, ${ }^{55}$ polymeric materials, ${ }^{54}$ biomolecules, ${ }^{92}$ and aqueous media ${ }^{93,94}$ have been successfully used. Materials are typically grown by electrodeposition or from solution based precursors using precipitation or metathesis reactions. An extensive review of templated approaches to nanorod construction has been recently published. ${ }^{56}$

One benefit of templated approaches is greater control over the morphology and size of the nanostructures. However, many factors need to be carefully considered for high quality nanorod growth. The template must be uniform and repetitious. Control of template orientation is highly desirable and no shrinkage or expansion should occur throughout the nanorod growth process. In many cases, the template must be removable after use. In the case of membranes, this is usually accomplished by destruction of the membrane by chemical reactions to which the nanorod is inert. Biological templates can be non-destructively removed by environmental changes such as $\mathrm{pH}$ or temperature.

Challenges for templated approaches include nanorod crystallinity and orientation. Correct positioning of the nanorods is vital for down-stream application development. This can be addressed by incorporating the template into the final device. The materials to be deposited 
must also by very pure. Uncontrolled impurities may perturb the crystal structure and result in multi-crystalline or amorphous materials or non-uniform optical and electronic properties. Conversely, this presents an ideal opportunity for controlled doping of the system.

In this experiment, a unique diffusive reaction system is used. Our growth system employs several principles of biomimetic growth. Biomimetic systems are those which imitate natural systems in function, growth, control strategies, or reactions. Although semiconductors are non-biogenic materials, several biomimetics principles have been demonstrated here. The reaction location is controlled by allowing the liquid and vapor to react at a membrane interface. The reactants are transported to the reaction location. The reaction and nanorod growth is confined by rigid polymer pores as well as thin membrane pores. Using biomimetic principles allows for greater control over reaction rate, location, and form of final products.

\subsection{Experimental}

Cadmium sulfate (99\%, Sigma Aldrich), zinc sulfide (99.9\%, Acros), and hydrochloric acid (environmental grade, Alfa Aesar) were used as received without further purification. Sylgard 184 silicone elastomer kit was mixed according to standard procedures. Nucleopore polycarbonate track etch (PCTE) membrane with pore diameters ranging from $0.05 \mu \mathrm{m}$ to 8 um was used as the template matrix.

\subsubsection{Setup}

Polydimethylsiloxane (PDMS) and curing agent were mixed at a ratio of $10: 1$ by weight. The mixture was thoroughly stirred to achieve an even distribution of curing agent. The mixture was degassed in a vacuum chamber to remove air and poured into a mold to a thickness of $\sim 1.5 \mathrm{~cm}$. The polymer was cured for 2 hours at $60{ }^{\circ} \mathrm{C}$, cooled, and cut into $2.5 \mathrm{~cm}^{2}$ pieces to fit into the reaction vessel. A $4 \mathrm{~mm}$ diameter hole was punched in the thick PDMS to direct the 
location of the reaction by providing an interface at which the reactants meet. The volume of this well is calculated to be $60 \mu \mathrm{l}$, though only $30 \mu \mathrm{l}$ of $\mathrm{CdSO}_{4}$ solution was placed in the well. Another size PDMS was also formed of the same dimensions, but only 50-100 $\mu \mathrm{m}$ thick. The thin PDMS is more permeable to hydrogen sulfide gas than thick PDMS. Porous polycarbonate track etch (PCTE) membrane was sized to fit the PDMS well and fixed to the thin PDMS first. This serves as the template for CdS nanorod and nanotube growth. One side of the membrane is smoother and therefore shinier. This side is placed against the thin PDMS to form a very strong and uniform seal. This also prevents growth of material between the membrane and PDMS interface. The membrane is then aligned over the PDMS well and the thin PDMS is firmly pressed against the thick PDMS to create a seal. The materials are assembled as shown in Figure 4-1. $\mathrm{CdSO}_{4}$ solution is loaded into the wells before the entire construct is inverted. By adding the liquid first, the high capillary forces maintain contact between the porous membrane and the aqueous cadmium solution. 


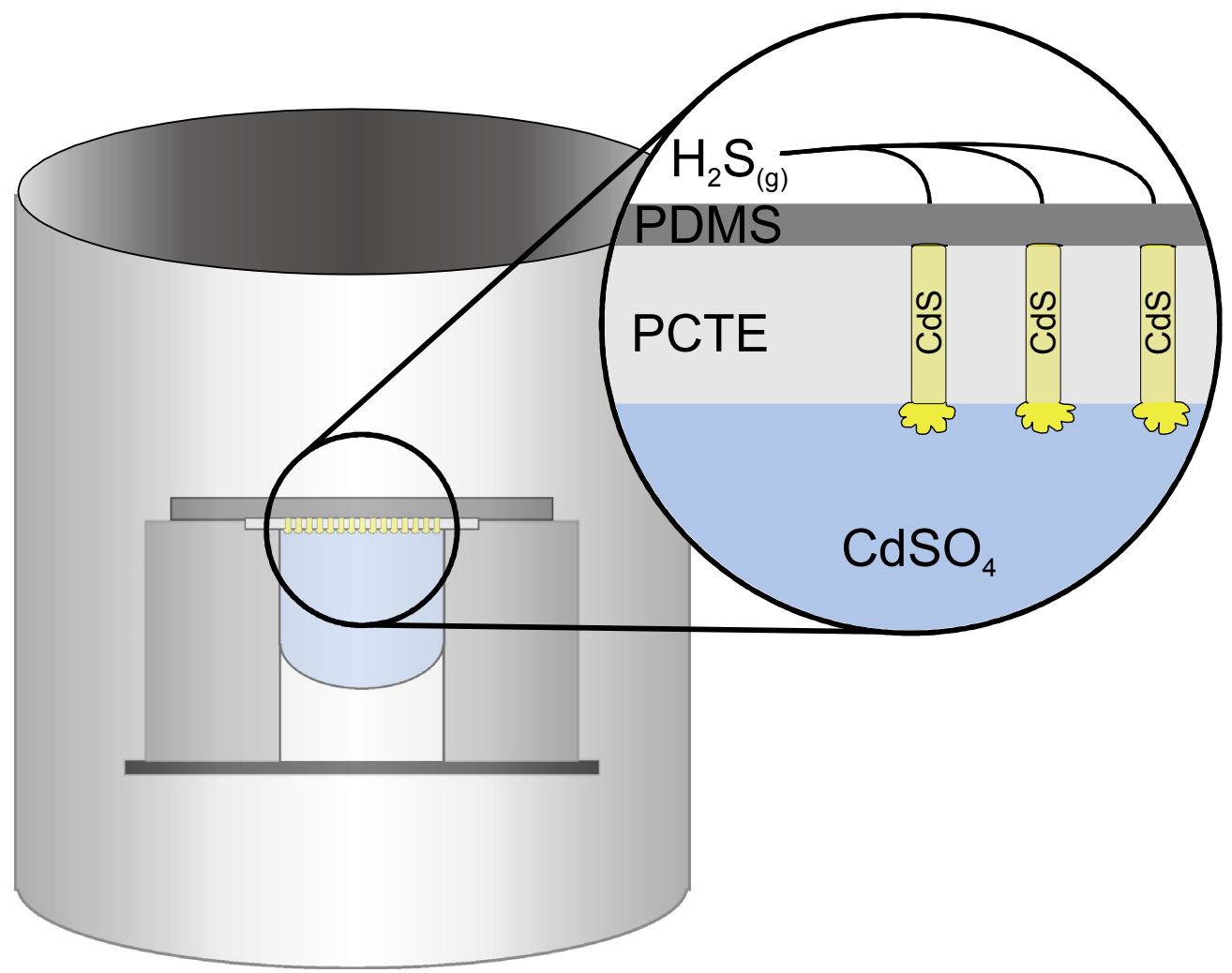

Figure 4-1. Schematic of CdS nanorod reaction and growth system.

Gaseous $\mathrm{H}_{2} \mathrm{~S}$ is generated as needed in a separate reaction vessel by adding $2 \mathrm{ml}$ of $30 \% \mathrm{HCl}\left(1.855 \times 10^{-2} \mathrm{~mol}\right)$ on $1 \mathrm{~g} \mathrm{ZnS} \mathrm{powder}\left(9.275 \times 10^{-3} \mathrm{~mol}\right)($ Scheme $4-1)$. This produces $\mathrm{H}_{2} \mathrm{~S}$ at a rate of approximately $0.0274 \mathrm{~cm}^{3} / \mathrm{s}$. As $\mathrm{H}_{2} \mathrm{~S}$ gas is generated, it flows into the reaction vessel where it reacts with $\mathrm{CdSO}_{4}$ to form CdS, an insoluble semiconductor (Scheme 4-2). The excess $\mathrm{H}_{2} \mathrm{~S}$ gas is removed by bubbling it through a solution of $\mathrm{NaOH}$, which produces sodium sulfide, a water-soluble byproduct.

\section{$\mathrm{ZnS}_{(\mathrm{s})}+2 \mathrm{HCl}_{(\mathrm{l})} \longrightarrow \mathrm{H}_{2} \mathrm{~S}_{(\mathrm{g})}+\mathrm{ZnCl}_{2 \text { (aq) }}$}

Scheme 4-1. Reaction used to produce hydrogen sulfide gas for subsequent reaction. 


\section{$\mathrm{H}_{2} \mathrm{~S}_{(\mathrm{g})}+\mathrm{CdSO}_{4(\mathrm{aq})} \longrightarrow \mathrm{CdS}_{(\mathrm{s})}+\mathrm{H}_{2} \mathrm{SO}_{4(\mathrm{aq})}$}

Scheme 4-2. Reaction that occurs in the membrane pores to produce CdS rods.

Figure 4-2 is an image of the actual reaction system used to produce semiconductor nanorods. PCTE functions as a template to control location and size of CdS formation consistent with biomimetic growth principles. ${ }^{95,96}$ Sulfur is provided by $\mathrm{H}_{2} \mathrm{~S}$ gas generated on demand. The rate of $\mathrm{H}_{2} \mathrm{~S}$ entering the reaction site is controlled by a thin ( $\left.50 \mu \mathrm{m}\right)$ PDMS layer which is somewhat permeable to gaseous $\mathrm{H}_{2} \mathrm{~S}$ molecules. ${ }^{97}$ Cadmium ions are supplied by aqueous cadmium sulfate. The high solubility of $\mathrm{CdSO}_{4}$ in water produces highly mobile $\mathrm{Cd}^{2+}$ ions. ${ }^{98}$ The membrane is coated with polyvinylpyrrolidone, a wetting agent that increases hydrophilicity of the membrane pores. This increases the amount of contact that the aqueous cadmium solution makes with the pores. The $\mathrm{H}_{2} \mathrm{~S}$ and $\mathrm{Cd}^{2+}$ ions interact in the membrane pores and form CdS - a highly insoluble semiconductor solid $\left(\mathrm{K}_{\mathrm{sp}}=1 \times 10^{-27}\right)$ After the material is formed, the membrane is immobilized in silver epoxy and the PCTE membrane is removed by oxygen plasma to produce freestanding CdS structures (Figure 4-3). 


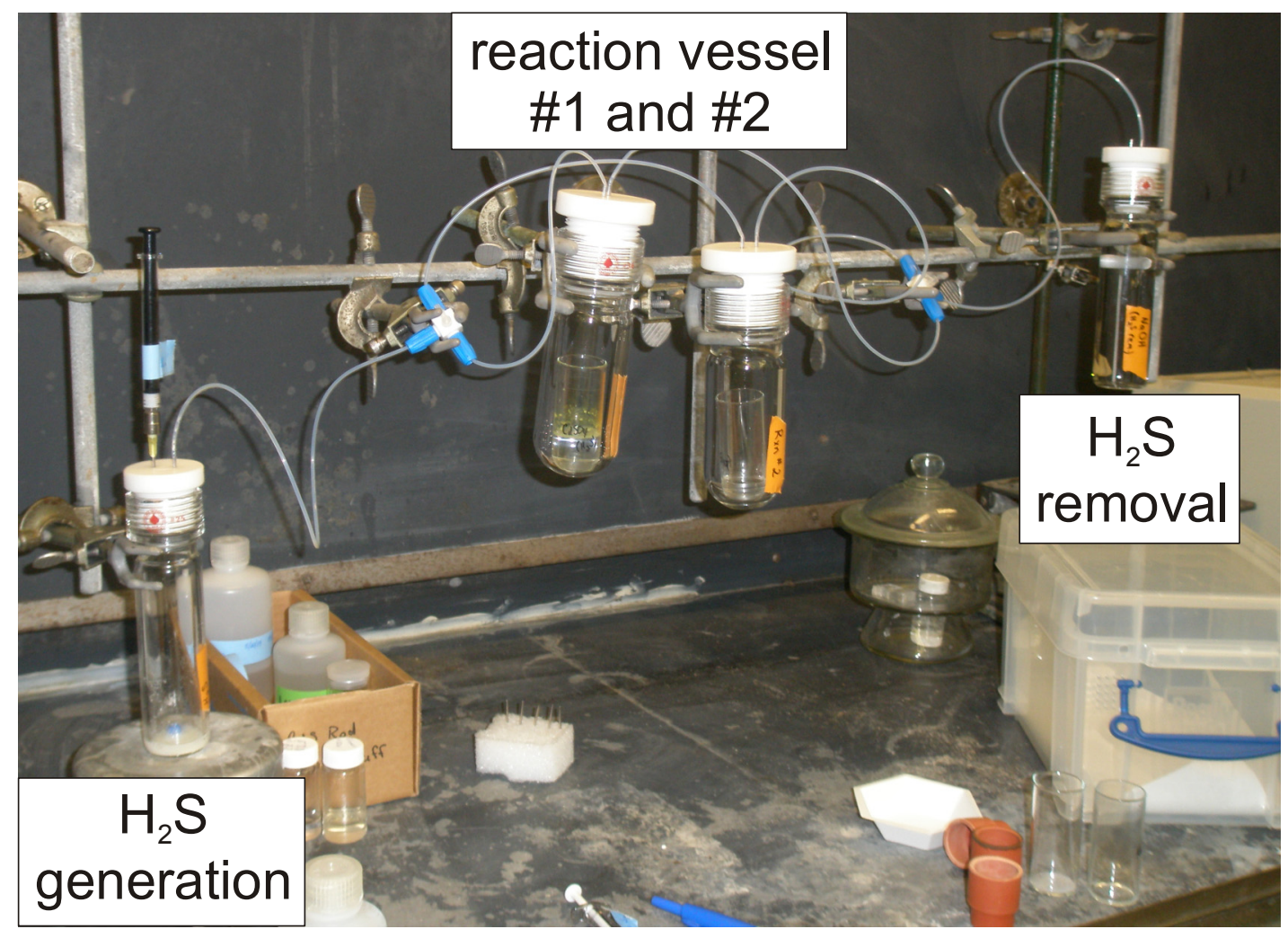

Figure 4-2. Photograph of actual reaction system used to produce CdS nanostructures. 

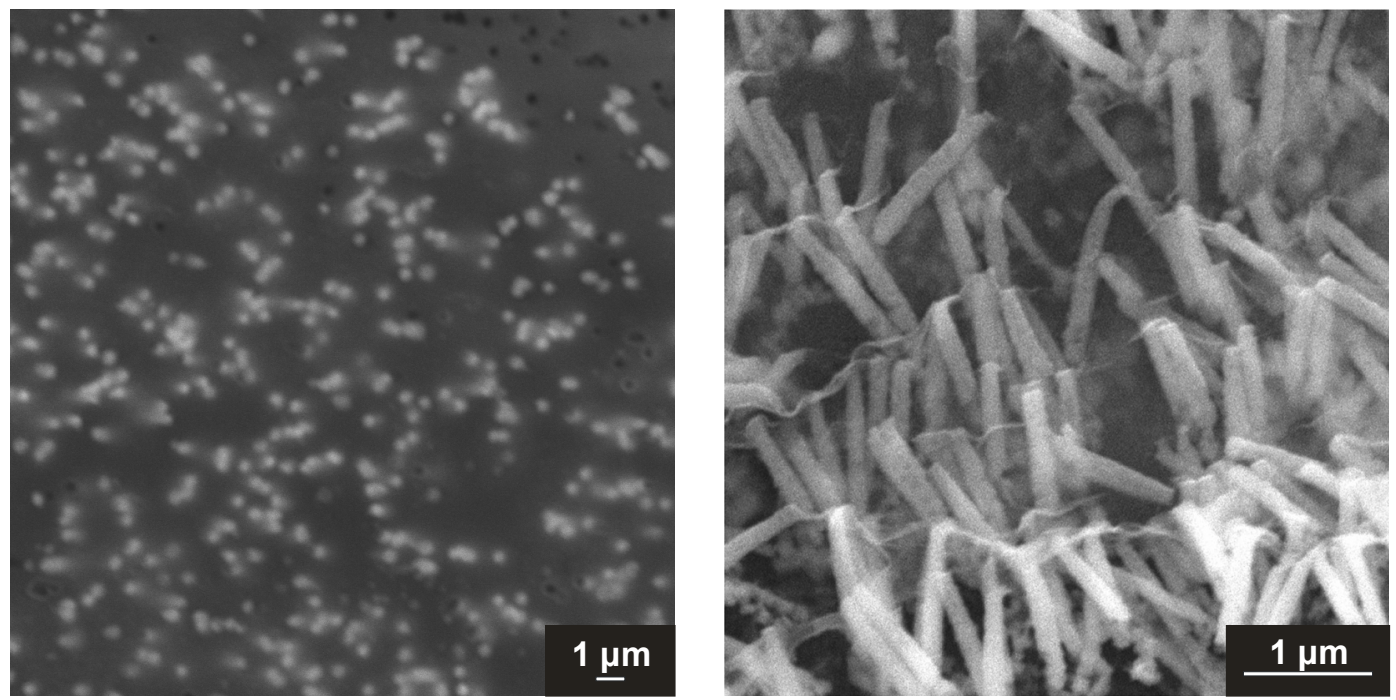

Figure 4-3. SEM images of CdS rods grown in PCTE membrane before (left) and after (right) plasma etching.

\subsubsection{Macro crystal growth}

To confirm that high quality cadmium sulfide material could be grown via this system and to determine appropriate concentrations and ratios of reactants, macroscale crystals were grown using thin PDMS placed on the PDMS wells without the PCTE templating membrane. This resulted in bulk CdS crystals grown directly on the thin PDMS surface. $\mathrm{CdSO}_{4}$ was dissolved in water to concentrations ranging from $5 \mathrm{M}$ to $0.01 \mathrm{M}$. The crystals were analyzed by optical microscopy, Raman spectroscopy, XRD, and EDS.

\subsubsection{Nanostructure growth}

Confined growth of anisotropic CdS structures was demonstrated by porous PCTE membrane with hole diameters ranging from $8 \mu \mathrm{m}$ to $0.05 \mu \mathrm{m}$. As described previously, the hydrophilic PCTE membrane was wetted by the $\mathrm{CdSO}_{4}$ solution and exposed to $\mathrm{H}_{2} \mathrm{~S}$ vapor. Size and morphology of the CdS nanostructures produced was related to $\mathrm{CdSO}_{4}$ concentration and length of exposure to $\mathrm{H}_{2} \mathrm{~S}$ environment. Rod and tube structures were analyzed 


\subsubsection{Characterization}

Raman spectroscopy: Raman spectroscopy was performed on a Bruker Senterra dispersive Raman microscope with a cooled CCD. Excitation wavelength was $532 \mathrm{~nm}$ at 20 $\mathrm{mW}$. Samples were analyzed directly on the thin PDMS substrates.

Electron microscopy: Scanning electron microscopy (SEM) and energy dispersive x-ray spectroscopy (EDS) were performed and electron dispersive x-ray spectra were obtained on a JEOL JSM6490-LVwith an INCA Energy SiLi detector. Macro crystal samples were prepared by depositing dry CdS crystals on carbon tape. Nanorod and tube samples were prepared by embedding the membrane in silver epoxy and then removing the membrane by plasma etching. $X$-ray diffraction spectroscopy: High angle x-ray diffraction (XRD) was performed on a custom-built machine using $\mathrm{Cu}$ Ka radiation with a wavelength of $0.15418 \mathrm{~nm}$. Samples were prepared by crushing CdS crystals and mixing the powder with grease on mica substrates.

\subsection{Results and Discussion}

CdS nanorods and nanotubes have been successfully synthesized in 0.1 to $8 \mu \mathrm{m}$ diameter pore templates (Figure 4-4). Biomimetic principles were effectively employed to confine the structure and growth of non-biogenic semiconductor materials. 

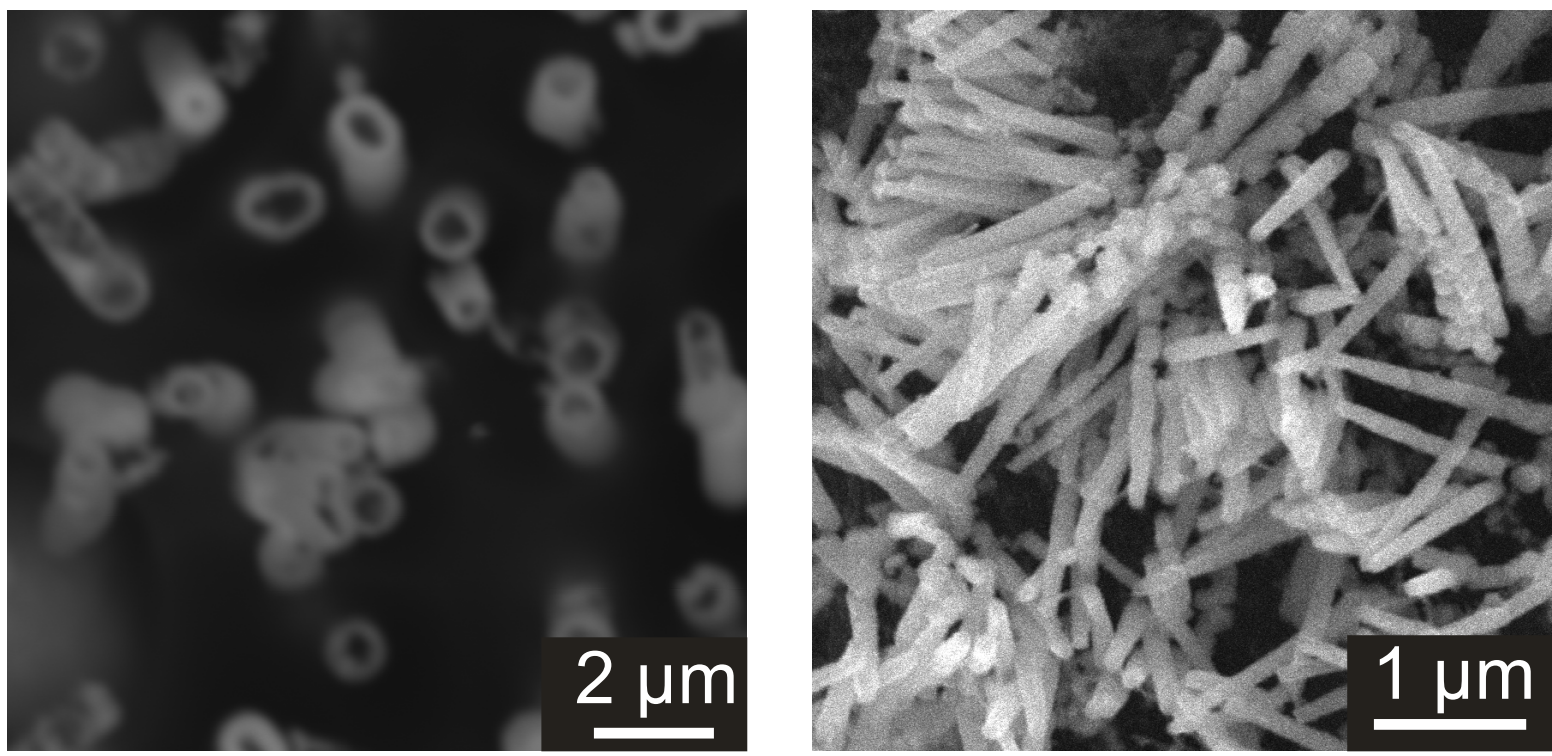

Figure 4-4. SEM images of CdS tubes (left) and rods (right).

Nanorods and nanowires composed of semiconducting materials have potential applications in communications, optical technologies, and integration with computational, sensing, and lab-on-a-chip devices. Existing synthetic strategies for semiconductor nanomaterials have required the use of potentially hazardous reactants, elevated temperatures, and complex synthetic schemes. These negative aspects have created significant challenges for further development and integration. New synthetic and assembly approaches are often necessary to facilitate novel materials and applications.

We have demonstrated a biomimetic synthesis for templated growth of nanomaterials at a liquid:solid interface under ambient conditions using low hazard reactants. We have successfully produced rods and tubes of various inorganic semiconductor materials in sizes ranging from nano- to microscale. The composition, crystalline structure, and spectroscopic properties of these materials have been extensively characterized. Of particular interest is the suitability of these rods for device applications such as biosensors and coherent light emitters. Characterization of the electronic properties of the semiconductor rods, as well as changes in 
apparent conductivity following surface binding events with small biological molecules is underway with a long-term goal of device development and other potential high-impact applications.

\subsubsection{Macro crystal growth and characterization}

Initially, bulk crystals were grown on PDMS without the membrane template to test our experimental design and to produce sufficient material for analysis and full characterization. Optical micrographs show brilliant yellow-orange crystals were produced (Figure 4-5). EDS confirms that these crystals are comprised of cadmium and sulfur (Figure 4-6). While CdS typically exists in nature in the hexagonal crystal structure, XRD of these materials clearly confirms that cubic CdS was produced (Figure 4-7). Further characterization by Raman shows the longitudinal optical (LO) vibrations expected for CdS (Figure 4-8).
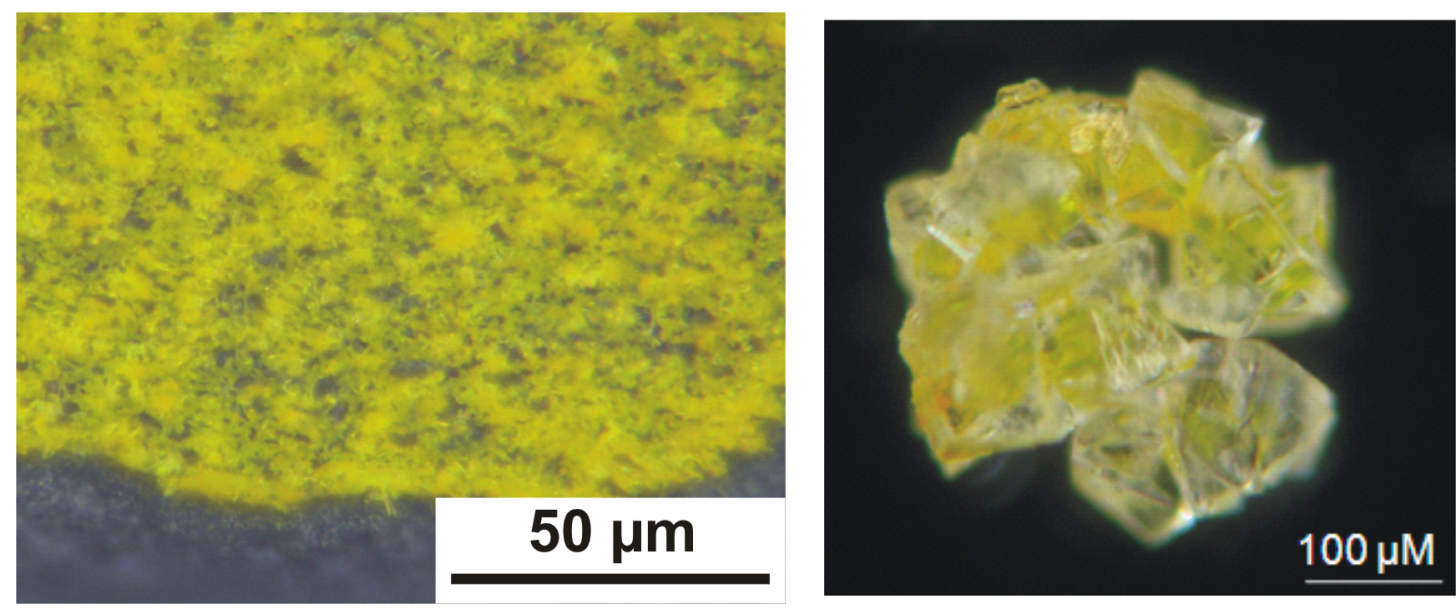

Figure 4-5. Optical micrographs of CdS macro crystals. 


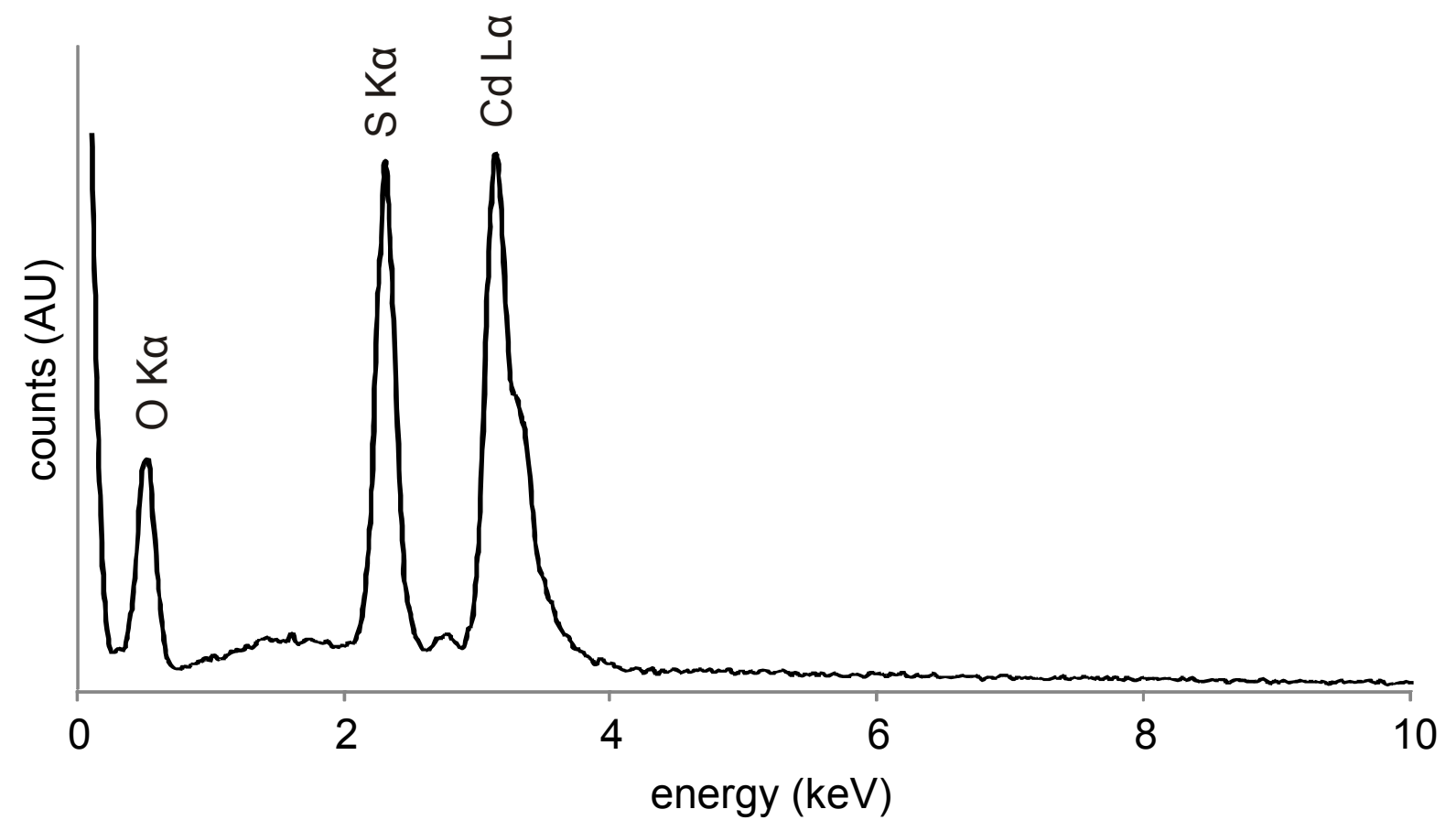

Figure 4-6. EDS spectrum of CdS crystals. 


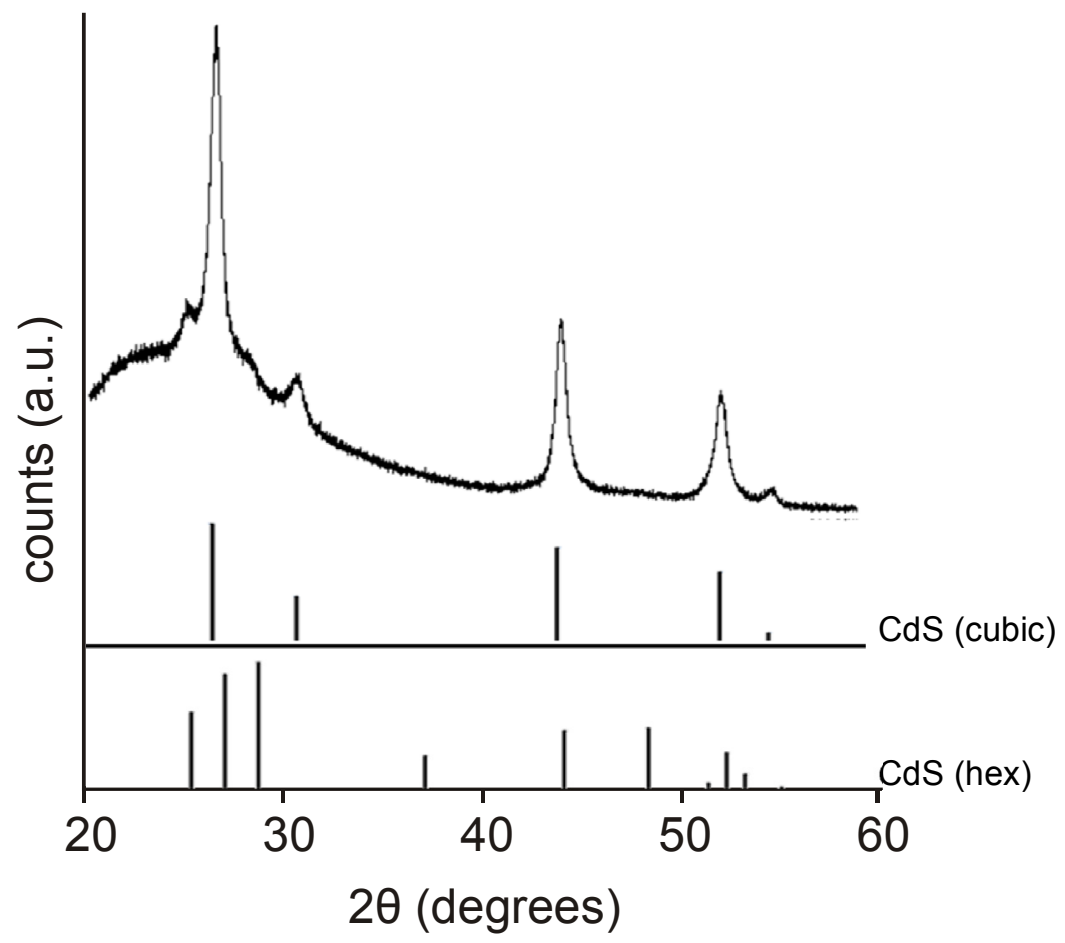

Figure 4-7. XRD spectrum of CdS crystals with comparison to CdS cubic and hexagonal peaks.

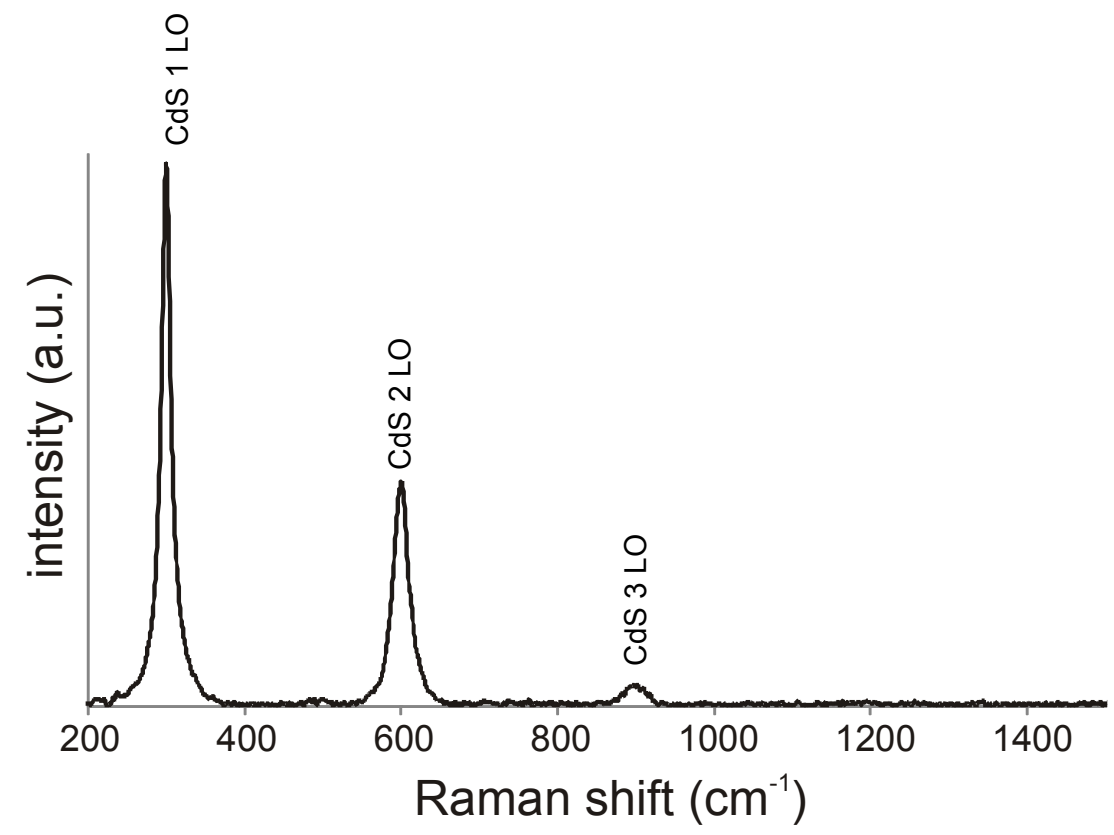

Figure 4-8. Raman spectrum of CdS crystals. 


\subsubsection{Nanostructure growth and characterization}

Using PCTE membranes of various sizes, rods and tubes were produced (Figure 4-4). The PCTE was successfully removed using oxygen plasma. It was confirmed by EDS spectroscopy that this process did not alter the chemical state of the CdS crystals or form cadmium oxide or other contaminants.

Aspect ratio and wall thickness were controlled by adjusting reaction time, cadmium ion concentration, and membrane pore size. Table 4-1 illustrates a few combinations of reaction conditions and the morphology of the resulting nanostructures as determined from SEM images.

At very long reaction times ( $>1$ hour), rods form in most pore sizes even at very low cadmium concentrations. At reaction times less than 1 hour, hollow nanotubes form. As expected, higher cadmium solution concentration or longer reaction time lead to increased wall thickness.

Table 4-1. Comparison of experimental conditions and observed nanostructures.

\begin{tabular}{ccccc}
$\begin{array}{c}{\left[\mathrm{CdSO}_{4}\right]} \\
(\mathrm{M})\end{array}$ & $\begin{array}{c}\text { rxn time } \\
(\mathrm{min})\end{array}$ & $\begin{array}{c}\text { pore diameter } \\
(\mu \mathrm{m})\end{array}$ & $\begin{array}{c}\text { wall thickness } \\
(\mu \mathrm{m})\end{array}$ & $\begin{array}{c}\text { approx. } \\
\text { aspect ratio }\end{array}$ \\
\hline 1.0 & 15 & 8.0 & 0.55 & 0.875 \\
5.0 & 15 & 8.0 & 1.26 & 0.875 \\
5.0 & 15 & 0.8 & 0.23 & 11.25 \\
1.0 & 45 & 0.8 & 0.32 & 11.25 \\
1.0 & 45 & 8.0 & 0.74 & 0.875 \\
5.0 & 45 & 8.0 & 1.68 & 0.875 \\
0.005 & 600.0 & 0.1 & rods & 60 \\
0.1 & 600.0 & 0.1 & rods & 60
\end{tabular}

The mechanism as established from time series reactions and SEM images suggests that the cadmium solution wets the pore sidewalls. When $\mathrm{H}_{2} \mathrm{~S}$ is introduced into the system, a ring of CdS quickly forms around the perimeter of the pore. As more cadmium solution is drawn 
into the pore from the PDMS well below, it forms small CdS crystallites also around the periphery. If allowed to react for enough time, these CdS crystallites aggregate to form solid rods. This is illustrated schematically with supporting SEM images in Figure 4-9. Calculations of pore size and $\mathrm{CdSO}_{4}$ solution concentration confirm that only enough cadmium ions are initially inside of the membrane pores to produce a few nanometer thick layer of cubic CdS. For more CdS to form inside of the pore, $\mathrm{Cd}^{2+}$ must be drawn up from the solution remaining in the PDMS well.
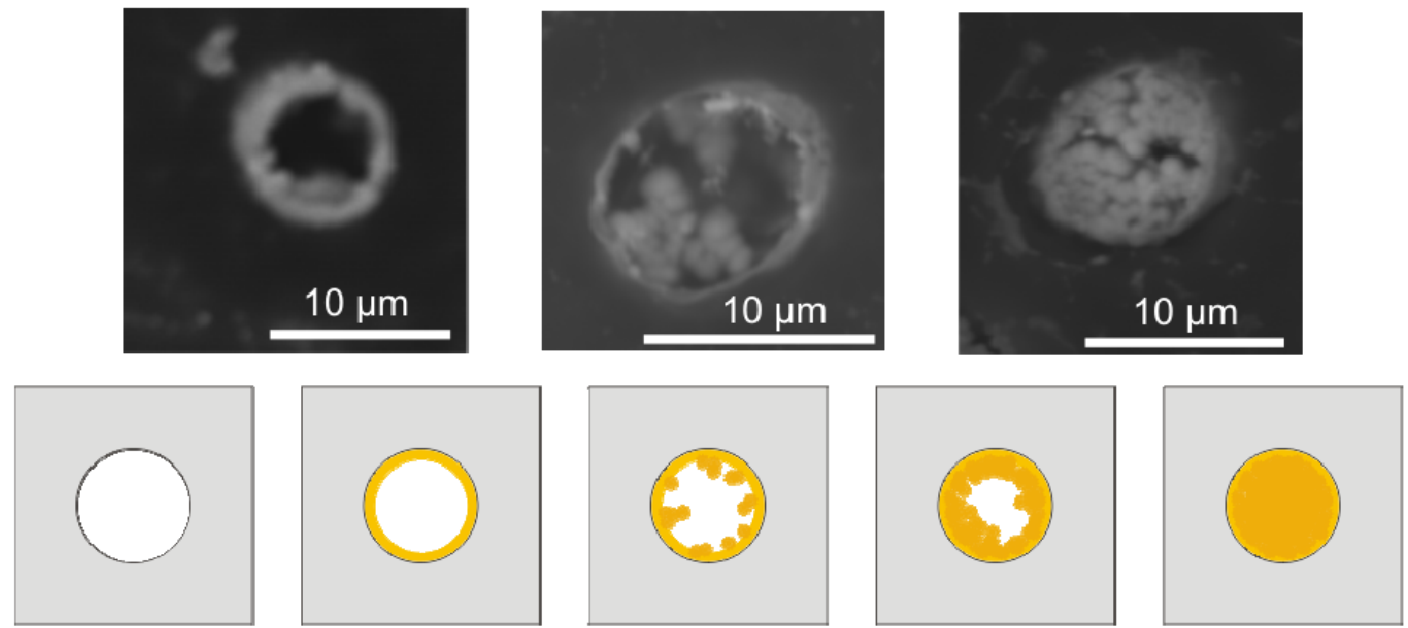

Top View
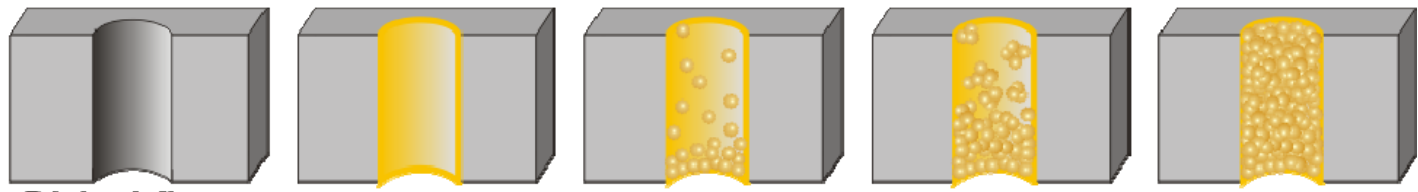

Side View

Figure 4-9. Schematic of CdS tube and rod formation supported with SEM images of actual growth.

\subsection{Conclusion}

A biomimetic system has been demonstrated to be applicable to the synthesis and control of non-biogenic semiconductor materials. This method offers a safe, simple growth of nano constructs with demonstrable control over size, morphology, and composition. 
Future research may utilize such nanorods and tubes to develop a biological sensor. Self-assembled monolayers of short chain acid or amine terminated ligands may be assembled on the nanorod surface to afford binding to biomolecules. Ligand/analyte pairs produce a selective binding response to proteins, DNA, or cells. Such binding events, when they occur at the surface of the nanorod, will alter the electronic properties of the nanorod when the ligand and analyte are bound. Such a signal produces a conductometric assay for binding events.

As nanostructures continue to grow in importance for technological, chemical, biomedical, and defense applications, the ability to control and fine-tune many aspects of the material becomes more important. With so many synthetic and control methods available, the mechanisms of growth are still being explored. Here we present a relatively simplistic, environmentally benign growth control that may be applied to an unknown variety of materials and morphologies. 


\section{References}

1. Keren, S.; Zavaleta, C.; Cheng, Z.; de la Zerda, A.; Gheysens, O.; Gambhir, S. S. Proceedings of the National Academy of Sciences 2008, 105, 5844-5849.

2. Cai, W.; Shin, D. W.; Chen, K.; Gheysens, O.; Cao, Q.; Wang, S. X.; Gambhir, S. S.; Chen, X. Nano Lett. 2006, 6, 669-676.

3. Chan, W. C.; Maxwell, D. J.; Gao, X.; Bailey, R. E.; Han, M.; Nie, S. Curr. Opin. Biotechnol. 2002, 13, 40-46.

4. Dubertret, B.; Skourides, P.; Norris, D. J.; Noireaux, V.; Brivanlou, A. H.; Libchaber, A. Science 2002, 298, 1759-1762.

5. Gao, X.; Chung, L. W. K.; Nie, S. In Quantum Dots 2007, p 135-145.

6. Jiang, W.; Singhal, A.; Zheng, J.; Wang, C.; Chan, W. C. W. Chem. Mater. 2006, 18, 48454854.

7. Vlasov, Y.; Yao, N.; Norris, D. Appl. Phys. Lett 1993, 62, 657.

8. Yoshie, T.; Shchekin, O.; Chen, H.; Deppe, D.; Scherer, A. Electron. Lett. 2002, 38, 967968.

9. Robel, I.; Subramanian, V.; Kuno, M.; Kamat, P. J. Am. Chem. Soc 2006, 128, 2385-2393.

10. Granström, M.; Petritsch, K.; Arias, A.; Lux, A.; Andersson, M.; Friend, R. Nature 1998, $395,257-260$.

11. Deb, P.; Kim, H.; Qin, Y.; Lahiji, R.; Oliver, M.; Reifenberger, R.; Sands, T. Nano Letters 2006, 6, 2893-2898.

12. Nicollian, E.; Tsu, R. Journal of Applied Physics 1993, 74, 4020.

13. Zhao, J.; Bardecker, J.; Munro, A.; Liu, M.; Niu, Y.; Ding, I.; Luo, J.; Chen, B.; Alex, K.; Ginger, D. Nano Letters 2006, 6, 463-467.

14. Bowers, M. J.; McBride, J. R.; Rosenthal, S. J. J. Am. Chem. Soc. 2005, 127, 1537815379.

15. Loss, D.; DiVincenzo, D. Phys. Rev. A 1998, 57, 120-126.

16. Michler, P.; Kiraz, A.; Becher, C.; Schoenfeld, W.; Petroff, P.; Zhang, L.; Hu, E.; Imamoglu, A. 2000; Vol. 290, p 2282-2285.

17. Routkevitch, D.; Tager, A.; Haruyama, J.; Almawlawi, D.; Moskovits, M.; Xu, J. IEEE Trans. Electron Devices 1996, 43, 1646-1658. 
18. Waks, E.; Inoue, K.; Santori, C.; Fattal, D.; Vuckovic, J.; Solomon, G.; Yamamoto, Y. Nature 2002, 420, 762.

19. Chu, M.; Liu, G. IEEE Transactions on Nanotechnology 2008, 7, 308-315.

20. Klostranec, J. M.; Xiang, Q.; Farcas, G. A.; Lee, J. A.; Rhee, A.; Lafferty, E. I.; Perrault, S. D.; Kain, K. C.; Chan, W. C. W. Nano Letters 2007.

21. Marinakos, S.; Chen, S.; Chilkoti, A. Anal. Chem 2007, 79, 5278-5283.

22. Huang, X.; Choi, Y. Sensors \& Actuators: B. Chemical 2007, 122, 659-671.

23. Chen, Y.; Nie, L.; Xue, X.; Wang, Y.; Wang, T. Appl. Phys. Lett. 2006, 88, 083105.

24. Godoi, R.; Kontozova, V.; Van Grieken, R. Atmos. Environ. 2006, 40, 1255-1265.

25. Colomban, P.; Etcheverry, M.; Asquier, M.; Bounichou, M.; Tournie, A. J. Raman Spectrosc. 2006, 37, 614-626.

26. Pradeep, T. Nano The Essentials; McGraw-Hill Education (India) Pvt Ltd, 2007.

27. Algar, W. R.; Krull, U. J. Langmuir 2008, 24, 5514-5520.

28. Becerra, L. R.; Murray, C. B.; Griffin, R. G.; Bawendi, M. G. The Journal of Chemical Physics 1994, 100, 3297-3300.

29. Berrettini, M. G.; Braun, G.; Hu, J. G.; Strouse, G. F. J. Am. Chem. Soc. 2004, 126, 70637070.

30. Boatman, E. M.; Lisensky, G. C. J. Chem. Ed. 2005, 82, 1697-1699.

31. Bullen, C. R.; Mulvaney, P. Nano Lett. 2004, 4, 2303-2307.

32. Chen, X.; Nazzal, A. Y.; Xiao, M.; Peng, Z. A.; Peng, X. Journal of Luminescence 2002, 97, 205-211.

33. Chen, Y.; Vela, J.; Htoon, H.; Casson, J. L.; Werder, D. J.; Bussian, D. A.; Klimov, V. I.; Hollingsworth, J. A. Journal of the American Chemical Society 2008, 130, 5026-5027.

34. Chin, P. T. K.; deMelloDonega, C.; vanBavel, S. S.; Meskers, S. C. J.; Sommerdijk, N. A. J. M.; Janssen, R. A. J. J. Am. Chem. Soc. 2007, 129, 14880-14886.

35. Katari, J. E. B.; Colvin, V. L.; Alivisatos, A. P. The Journal of Physical Chemistry 1994, 98, 4109-4117.

36. Kippeny, T. C.; Bowers, M. J.; II; Dukes, A. D.; III; McBride, J. R.; Orndorff, R. L.; Garrett, M. D.; Rosenthal, S. J. The Journal of Chemical Physics 2008, 128, 084713.

37. Kirchner, C.; Liedl, T.; Kudera, S.; Pellegrino, T.; Javier, A. M.; Gaub, H. E.; Stölzle, S.; Fertig, N.; Parak, W. J. Nano Letters 2005, 5, 331-338. 
38. Leatherdale, C. A.; Woo, W.-K.; Mikulec, F. V.; Bawendi, M. G. The Journal of Physical Chemistry B 2002, 106, 7619-7622.

39. Peng, X.; Manna, L.; Yang, W.; Wickham, J.; Scher, E.; Kadavanich, A.; Alivisatos, A. P. Nature 2000, 404, 59-61.

40. Peng, X.; Peng, Z. A.; Qu, L. Nano Letters 2001, 1, 333-337.

41. Peng, X.; Schlamp, M. C.; Kadavanich, A. V.; Alivisatos, A. P. Journal of the American Chemical Society 1997, 119, 7019-7029.

42. Peng, Z. A.; Peng, X. J. Am. Chem. Soc. 2001, 123, 183-184.

43. Qu, L.; Peng, X. J. Am. Chem. Soc. 2002, 124, 2049-2055.

44. Reiss, P.; Bleuse, J.; Pron, A. Nano Letters 2002, 2, 781-784.

45. Reiss, P.; Carayon, S.; Bleuse, J.; Pron, A. Synthetic Metals 2003, 139, 649-652.

46. Talapin, D. V.; Rogach, A. L.; Kornowski, A.; Haase, M.; Weller, H. Nano Letters 2001, 1, 207-211.

47. Talapin, D. V.; Shevchenko, E. V.; Murray, C. B.; Kornowski, A.; Forster, S.; Weller, H. Journal of the American Chemical Society 2004, 126, 12984-12988.

48. Vasiliev, R.; Vinogradov, V.; Dorofeev, S.; Kozyrev, S.; Kucherenko, I.; Novikova, N. Physics of the Solid State 2007, 49, 547-551.

49. Yu, W. W.; Qu, L.; Guo, W.; Peng, X. Chem. Mater. 2003, 15, 2854-2860.

50. Nirmal, M.; Brus, L. Accounts of Chemical Research 1999, 32, 407-414.

51. Soloviev, V. N.; Eichhofer, A.; Fenske, D.; Banin, U. Journal of the American Chemical Society 2000, 122, 2673-2674.

52. Xin, S.; Wang, P.; Yin, A.; Kim, C.; Dobrowolska, M.; Merz, J.; Furdyna, J. Appl. Phys. Lett. 1996, 69, 3884.

53. Schikora, D.; Schwedhelm, S.; As, D.; Lischka, K.; Litvinov, D.; Rosenauer, A.; Gerthsen, D.; Strassburg, M.; Hoffmann, A.; Bimberg, D. Appl. Phys. Lett. 2000, 76, 418.

54. Zhan, J.; Yang, X.; Wang, D.; Li, S.; Xie, Y.; Xia, Y.; Qian, Y. Advanced Materials 2000, 12.

55. Routkevitch, D.; Bigioni, T.; Moskovits, M.; Xu, J. M. J. Phys. Chem. 1996, 100, 1403714047.

56. Hurst, S. J.; Payne, E. K.; Qin, L.; Mirkin, C. A. Angewandte Chemie International Edition 2006, 45, 2672-2692.

57. Murray, C. B.; Noms, D. J.; Bawendi, M. G. J. Am. Chem. Soc. 1993, 115, 8706-8715. 
58. Manna, L.; Scher, E. C.; Alivisatos, A. P. Journal of the American Chemical Society 2000, 122, $12700-12706$.

59. Barrelet, C. J.; Wu, Y.; Bell, D. C.; Lieber, C. M. Journal of the American Chemical Society 2003, 125, 11498-11499.

60. Gudiksen, M.; Lieber, C. Growth 2000, 127, 849-857.

61. Hu, J.; Odom, T.; Lieber, C. Accounts of Chemical Research 1999, 32, 435-446.

62. Ravindran, S.; Chaudhary, S.; Colburn, B.; Ozkan, M.; Ozkan, C. Nano Letters 2003, 3, 447-453.

63. Bruchez, M.; Hotz, C. Quantum dots: applications in biology; Humana Press, 2007.

64. Dabbousi, B. O.; Rodriguez-Viejo, J.; Mikulec, F. V.; Heine, J. R.; Mattoussi, H.; Ober, R.; Jensen, K. F.; Bawendi, M. G. J. Phys. Chem. B 1997, 101, 9463-9475.

65. Derfus, A. M.; Chan, W. C. W.; Bhatia, S. N. Nano Letters 2004, 4, 11-18.

66. Tsay, J. M.; Michalet, X. Chem. Biol. 2005, 12, 1159-1160.

67. Anastas, P.; Warner, J. Green Chemistry: Theory and Practice; Oxford University Press, USA, 2000.

68. Peng, X. Chemistry - A European Journal 2002, 8, 334-339.

69. Gerion, D.; Pinaud, F.; Williams, S. C.; Parak, W. J.; Zanchet, D.; Weiss, S.; Alivisatos, A. P. J. Phys. Chem. B 2001, 105, 8861-8871.

70. Christian, P.; O'Brien, P. J. Mater. Chem. 2008, 18, 1689-1693.

71. Datta, A.; Panda, S. K.; Chaudhuri, S. The Journal of Physical Chemistry C 2007, 111, 17260-17264.

72. Gu, F. X.; Karnik, R.; Wang, A. Z.; Alexis, F.; Levy-Nissenbaum, E.; Hong, S.; Langer, R. S.; Farokhzad, O. C. NanoToday 2007, 2, 14-21.

73. Michalet, X.; Pinaud, F. F.; Bentolila, L. A.; Tsay, J. M.; Doose, S.; Li, J. J.; Sundaresan, G.; Wu, A. M.; Gambhir, S. S.; Weiss, S. Science 2005, 307, 538-544.

74. Peng, X.; Wickham, J.; Alivisatos, A. Journal of the American Chemical Society 1998, 120, 5343-5344.

75. Brus, L. E. J. Chem. Phys. 1984, 80, 4403-4409.

76. Lifshitz, E.; Brumer, M.; Kigel, A.; Sashchiuk, A.; Bashouti, M.; Sirota, M.; Galun, E.; Burshtein, Z.; Quang, A. Q. L.; Ledoux-Rak, I.; Zyss, J. J. Phys. Chem. B 2006, 110, 25356-25365.

77. Hines, M.; Guyot-Sionnest, P. J. Phys. Chem. 1996, 100, 468-471. 
78. Murray, C.; Kagan, C.; Bawendi, M. Annual Review of Materials Science 2000, 30, 545610.

79. Zhelev, Z.; Ohba, H.; Bakalova, R.; Jose, R.; Fukuoka, S.; Nagase, T.; Ishikawaa, M.; Baba, Y. Chem Commun 2005, 1980-1982.

80. Bruchez Jr, M.; Moronne, M.; Gin, P.; Weiss, S.; Alivisatos, A. Science 1998, 281, 2013.

81. Helfferich, F. Nature 1961, 189, 1001-1002.

82. Gao, X.; Chung, L.; Nie, S. In Quantum Dots 2007; Vol. 374, p 135.

83. Shen, H.; Jawaid, A.; Snee, P. ACS Nano 2009, 12142-12150.

84. Wang, C.-W.; Moffitt, M. G. Langmuir 2004, 20, 11784-11796.

85. Shen, H.; Jawaid, A. M.; Snee, P. T. ACS Nano 2009, 0.

86. Wilbur, J. L.; Kumar, A.; Kim, E.; Whitesides, G. M. Advanced Materials 1994, 6, 600-604.

87. Reya, T.; Clevers, H. Nature 2005, 434, 843-850.

88. Hu, J.; Li, L.-S.; Yang, W.; Manna, L.; Wang, L.-W.; Alivisatos, A. P. Science 2001, 292, 2060-2063.

89. Duan, X.; Huang, Y.; Cui, Y.; Wang, J.; Lieber, C. Nature 2001, 409, 66.

90. Klimov, V. I.; Mikhailovsky, A. A.; Xu, S.; Malko, A.; Hollingsworth, J. A.; Leatherdale, C. A.; Eisler, H. J.; Bawendi, M. G. Science 2000, 290, 314-317.

91. Kortan, A. R.; Hull, R.; Opila, R. L.; Bawendi, M. G.; Steigerwald, M. L.; Carroll, P. J.; Brus, L. E. J. Am. Chem. Soc. 1990, 112, 1327-1332.

92. Braun, E.; Eichen, Y.; Sivan, U.; Ben-Yoseph, G. Nature 1998, 391, 775.

93. Nanda, J.; Sapra, S.; Sarma, D.; Chandrasekharan, N.; Hodes, G. Chem. Mater 2000, 12, 1018-1024.

94. Simmons, B. A.; Li, S. C.; John, V. T.; McPherson, G. L.; Bose, A.; Zhou, W. L.; He, J. B. Nano Letters 2002, 2, 263-268.

95. Mann, S. Biomimetic materials chemistry 1996, 1-37.

96. Collier, J.; Messersmith, P. Annual Review of Materials Research 2001, 31, 237-263.

97. Stern, S.; Bhide, B. Journal of Applied Polymer Science 1989, 38.

98. Alberty, R. A.; King, E. L. Journal of the American Chemical Society 1951, 73, 517-523.

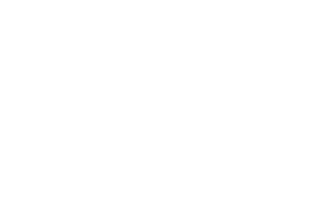

